\title{
Topological Sensitivity Analysis and Kohn-Vogelius Formulation for Detecting a Rigid Inclusion in an Elastic Body
}

\author{
Mourad Hrizi
}

\begin{abstract}
Our main interest in this work is to detect a rigid inclusion immersed in an isotropic elastic body $\Omega$ from a single pair of Cauchy data on $\partial \Omega$ in two dimensions. We want to completely characterize the unknown rigid inclusion, namely, the shape and the location of inclusion. The idea is to rewrite the inverse problem as an optimization problem, where an energy like functional is minimized with respect to the presence of a small inclusion. A topological sensitivity analysis is derived for an energy like functional. We proposed a non-iterative reconstruction algorithm based on the topological gradient concept. The unknown rigid inclusion is defined by a level curve of a scalar function. The proposed numerical approach is very robust with respect to noisy data. Finally, in order to show the efficiency and accuracy of the proposed algorithm, we present some numerical results.
\end{abstract}

\section{Introduction}

The detection of inclusions and/or cavities using the over-determined boundary data is a classical inverse problem that arises in nondestructive testing for damage assessment of mechanical specimens which are possible defective due to the presence of interior void (rigid inclusions and cavities) induced during the manufacturing process. There have been numerous methods for void detection $3,8,10,15,20,21,37,41,43,44,52$.

In this paper, we address the problem of nondestructing testing: to reconstruct the location and shape of a rigid inclusion (defined by a Dirichlet boundary condition) immersed in an elastic body $\Omega$ (represented by a bounded domain in $\mathbb{R}^{2}$ ) by applying a traction field at the boundary $\partial \Omega$ and by measuring the induced displacement field on the boundary $\partial \Omega$. The displacement field satisfies the elasticity framework. This inverse problem is known to be severely ill-posed and nonlinear. Alves and Martins in 9] solved this inverse problem and proposed an approach based on the method of fundamental solutions (MFS). They established a numerical scheme that connects the MFS. While Karageorghis et al. 39, 40 proposed a regularization approach relies on the minimization of a nonlinear least-squares

Received March 22, 2019; Accepted July 15, 2019.

Communicated by Jenn-Nan Wang.

2010 Mathematics Subject Classification. 49Q10, 35R30, 49Q12, 74P15.

Key words and phrases. shape optimization, topological optimization, topological sensitivity analysis, linear elasticity, Kohn-Vogelius functional. 
functional, penalized with respect to both the MFS and the derivative of the radial polar coordinates describing the position of the rigid inclusions or cavities. Then Shifrin and Shushpannikov in [55] determined a spheroidal cavity or rigid inclusion in an elastic solid by applying an analytic approach based on the reciprocity gap functional (RGF). Moreover, the reconstruction of inclusion can also be analyzed in a different framework in terms of a change in the elastic material properties [4, 48, 50].

Here, to reconstruct a rigid inclusion inside an elastic body, we propose an alternative reconstruction approach combining the advantages of the Kohn-Vogelius formulation 42 and the topological sensitivity analysis method [51]. The idea is to reformulate the inverse problem into a shape optimization one, where the rigid inclusion is the unknown variable. The method relies on the minimization of the so-called Kohn-Vogelius type functional, which measures the difference between the solutions of two auxiliary problems. To minimize this functional, we apply the topological sensitivity analysis. The main advantage of this reconstruction method is that it provides fast and accurate results for detection.

The main contribution of this paper concerns the theoretical and numerical aspects. In the theoretical part, we have derived a topological sensitivity analysis for the linear elasticity problem with respect to the insertion of a small inclusion in the domain $\Omega$ with a Dirichlet condition on the boundary of the inclusion. The obtained results are based on a rigorous and simplified mathematical analysis valid for a large class of shape functions. This method allows us to perform the topological sensitivity analysis without using the truncation technique proposed in [29]. In the numerical part, we propose a non-iterative algorithm for reconstructing an inclusion. The efficiency of the proposed algorithm is illustrated by some numerical examples. Particularly, we test the influence of some parameters in our algorithm such as the shape, location, size and the number of the inclusions.

This paper is summarized and rounded up as follows. Section 2 contains some notation and presents the direct and inverse problems. The proposed approach to solve the considered inverse problem is described in Section 3. In Section 4 , we derive a topological asymptotic analysis with respect to the creation of a small rigid inclusion. Then, we propose a non-iterative algorithm. The efficiency and accuracy of the proposed algorithm are illustrated by some numerical results provided in Section 5. The sixth section is devoted to a conclusion, whereas the closing section records some definitions and preliminary results.

\section{The problem setting}

\subsection{Notations}

Let us introduce some notations which will be useful in what follows. For an open and bounded domain $\Omega \subset \mathbb{R}^{2}$, we denote by $\mathrm{W}^{m, p}(\Omega)$ and $H^{s}(\Omega):=\left[H^{s}(\Omega)\right]^{2}$ the usual 
Lebesgue and Sobolev spaces. Moreover, we denote by $\|\cdot\|_{1, \Omega}$ the norm $\|\cdot\|_{H^{1}(\Omega)}$ and $|\cdot|_{1, \Omega}$ the semi-norm $\|\cdot\|_{H^{1}(\Omega)}$ defined in $(7.1)$. We also denote by $\|\cdot\|_{1 / 2, \partial \Omega}$ the norm $\|\cdot\|_{H^{1 / 2}(\partial \Omega)}$ and $\|\cdot\|_{-1 / 2, \partial \Omega}$ the norm $\|\cdot\|_{H^{-1 / 2}(\partial \Omega)}$. We represent the duality product between $H^{-1 / 2}(\partial \Omega)$ and $H^{1 / 2}(\partial \Omega)$ by the notation $\langle\cdot, \cdot\rangle_{-1 / 2,1 / 2, \partial \Omega}$. Finally, we define an inner product for matrices by $M: N=\sum_{i, j=1}^{2} M_{i j} N_{i, j}$ for $M, N \in \mathbb{R}^{2 \times 2}$; the associated norm is $|M|=\sqrt{M: M}$. The corresponding inner product on $L^{2}(\Omega)^{2 \times 2}$ is

$$
\langle M, N\rangle_{L^{2}(\Omega)^{2 \times 2}}=\int_{\Omega} M(x): N(x) \mathrm{d} x \quad \text { for } M, N \in L^{2}(\Omega)^{2 \times 2} .
$$

\subsection{The direct problem}

Let $\Omega$ be an open and bounded domain of $\mathbb{R}^{2}$ with smooth boundary $\partial \Omega$, occupied by a linear elastic material. Inside the domain $\Omega$, we assume the existence of a simply connected subdomain $\mathscr{B}^{*} \subset \Omega$ (rigid inclusion) with boundary $\partial \mathscr{B}^{*}$ of class $C^{1}$ such that $\operatorname{dist}\left(\partial \mathscr{B}^{*}, \partial \Omega\right)>0$ (see Figure 2.1).

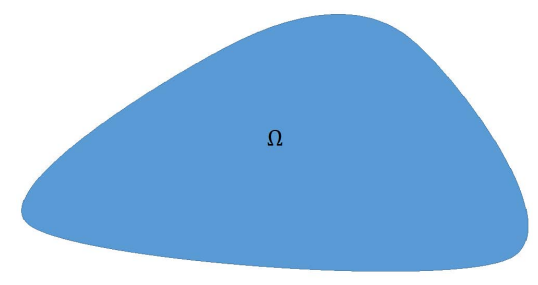

(a)

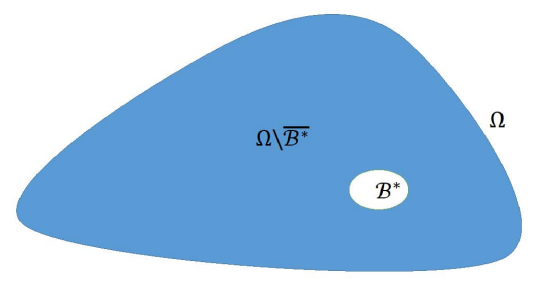

(b)

Figure 2.1: (a) Domain $\Omega$ without inclusion and (b) Domain $\Omega$ with inclusion $\mathscr{B}^{*}$.

The forward linear elastic problem is, therefore, given by

$$
\begin{cases}-\operatorname{div} \sigma(\psi)=0 & \text { in } \Omega \backslash \overline{\mathscr{B}^{*}} \\ \sigma(\psi) \mathbf{n}=\varphi & \text { on } \partial \Omega, \\ \psi=0 & \text { on } \partial \mathscr{B}^{*},\end{cases}
$$

where $\varphi \not \equiv 0$ is a given traction acting on the boundary $\partial \Omega$ and $\mathbf{n}$ denotes the outward unit normal vector to the boundary $\partial \Omega$. The vector $\psi$ denotes the displacement field, $\sigma=\left(\sigma_{i j}\right)_{1 \leq i, j \leq 2}$ is the associated Cauchy stress tensor and $e=\left(e_{i j}\right)_{1 \leq i, j \leq 2}$ is the linearized strain given by

$$
e_{i j}(\psi)=\frac{1}{2}\left(\frac{\partial \psi_{i}}{\partial x_{j}}+\frac{\partial \psi_{j}}{\partial x_{i}}\right), \quad 1 \leq i, j \leq 2
$$

Note that $\sigma$ and $e$ are related by the Hooke constitutive law [26], the medium being 
assumed to be homogeneous and isotropic. Therefore,

$$
\sigma_{i j}(\psi)=2 \mu e_{i j}(\psi)+\lambda \delta_{i j} \sum_{k=1}^{2} e_{k k}(\psi), \quad 1 \leq i, j \leq 2 .
$$

Above, $\delta_{i j}$ is the Kronecker symbol and $\mu, \lambda$ are the Lamé coefficients related to Young's modulus $E$ and the Poisson's ratio $\nu$ via

$$
\mu=\frac{E}{2(1+\nu)} \quad \text { and } \quad \lambda=\frac{E \nu}{(1+\nu)(1-2 \nu)} .
$$

For the physical meaning of these parameters, see 38 .

\subsection{The inverse problem}

We define the class of admissible geometries

$$
\begin{aligned}
& \mathrm{V}_{\text {ad }}:=\left\{\mathscr{B} \subset \subset \Omega: \mathscr{B} \text { is a simply connected open set, } \partial \mathscr{B} \text { is of class } C^{1},\right. \\
&\left.\operatorname{dist}(x, \partial \Omega) \geq \rho_{0}>0, \forall x \in \mathscr{B} \text { and } \Omega \backslash \overline{\mathscr{B}} \text { is connected }\right\}
\end{aligned}
$$

with $\rho_{0}$ being a small fixed parameter.

In this work, we focus on the reconstruction of the location and the shape of an unknown rigid inclusion $\mathscr{B}^{*} \in \mathrm{V}_{\mathrm{ad}}$ immersed in the elastic body $\Omega$ from a single pair of displacement and surface traction data on the boundary of $\Omega$. Therefore, the inverse problem that we consider here can be formulated as follows:

- Assume having an over-determined data on $\partial \Omega$ such as

$\circ \varphi \in H^{-1 / 2}(\partial \Omega)$ is a given traction field:

$$
\sigma(\psi) \mathbf{n}=\varphi \quad \text { on } \partial \Omega
$$

$\circ \mathscr{U}_{d} \in H^{1 / 2}(\partial \Omega)$ is a measured displacement field:

$$
\psi=\mathscr{U}_{d} \quad \text { on } \partial \Omega
$$

- Reconstruct the unknown rigid inclusion $\mathscr{B}^{*} \in \mathrm{V}_{\text {ad }}$ such that the displacement field $\psi$ in the presence of the rigid inclusion $\mathscr{B}^{*}$ satisfies the following over-determined boundary value problem

$$
\begin{cases}-\operatorname{div} \sigma(\psi)=0 & \text { in } \Omega \backslash \overline{\mathscr{B}^{*}} \\ \sigma(\psi) \mathbf{n}=\varphi & \text { on } \partial \Omega, \\ \psi=\mathscr{U}_{d} & \text { on } \partial \Omega, \\ \psi=0 & \text { on } \partial \mathscr{B}^{*} .\end{cases}
$$


The data $\mathscr{U}_{d}$ for which this problem has a solution $\psi$ is said to be compatible.

The theoretical aspect of the over-determined problem $(2.2)$ has been the subject of various researcher's works. Particularly, one can consult [9,49 for identifiability and stability results. Alves and Martins in [9] discussed the question of the identification of inclusions/cavities in an elastic body, using a single boundary measurement. Therefore, we have the following uniqueness theorem from [9, Theorem 3]:

Theorem 2.1 (Uniqueness). Let $\overline{\mathscr{B}^{*}} \subset \Omega \subset \mathbb{R}^{2}$ be bounded and simply connected domains with $\mathscr{C}^{1}$-smooth boundaries such that the domain $\Omega \backslash \overline{\mathscr{B}^{*}}$ is connected. Let also the Neumann and Dirichlet data $\varphi \in H^{-1 / 2}(\partial \Omega)$ and $\mathscr{U}_{d} \in H^{1 / 2}(\partial \Omega)$. If $\mathscr{U}_{d} \neq \equiv 0$ then a single pair of Cauchy data $\left(\varphi, \mathscr{U}_{d}\right)$ determines uniquely the displacement field $\psi^{*} \in H^{1}\left(\Omega \backslash \overline{\mathscr{B}^{*}}\right)$ and the rigid inclusion $\mathscr{B}^{*}$ satisfying the inverse problem is given by 2.2 .

On the other hand, Morassi and Rosset in [49] established the uniqueness and a conditional stability estimate of log-log type in determining a rigid inclusion inside an isotropic elastic body, we have the following stability theorem from 49, Theorem 2.5]:

Theorem 2.2 (Stability). Let $\mathscr{B}_{i}, i=1,2$, be two open subsets in the class of admissible geometries $\mathrm{V}_{\mathrm{ad}}$. Moreover, let $\Sigma$ be an open portion of $\partial \Omega$. Let $\psi_{i} \in H^{1}(\Omega \backslash \overline{\mathscr{B}}$ ) be the solution to 2.1), and let $\varphi \in H^{1 / 2}(\partial \Omega)$ when $\mathscr{B}=\mathscr{B}_{i}, i=1,2$. If for a given $\varepsilon>0$ we have

$$
\min _{r \in \mathscr{R}}\left\|\left(\psi_{1}-\psi_{2}\right)-r\right\|_{L^{2}(\Sigma)} \leq \rho_{0}^{1 / 2} \varepsilon
$$

then

$$
d_{\mathscr{H}}\left(\partial \mathscr{B}_{1}, \partial \mathscr{B}_{2}\right) \leq \rho_{0} \omega\left(\frac{\varepsilon}{\rho_{0}^{1 / 2}\|\varphi\|_{H^{-1 / 2}(\partial \Omega)}}\right)
$$

where $\mathscr{R}$ denotes the linear space of the infinitesimal rigid displacements and $\omega$ is an increasing continuous function on $[0, \infty)$ which satisfies

$$
\omega(t) \leq C(\log |\log t|)^{-\iota} \quad \text { for all } t, 0<t<e^{-1},
$$

and $C, \iota, C>0,0<\iota \leq 1$, are constants only depending on the a priori data.

From the above Theorems 2.1 and 2.2, we deduce that the solution of the inverse problem 2.2 is unique and stable.

In order to solve the reconstruction problem 2.2 , we rephrase the inverse problem under consideration into an optimal design one. We propose a reconstruction approach based on the Kohn-Vogelius formulation and the topological sensitivity analysis method. 


\section{The reconstruction method}

Our reconstruction approach is based on two main steps. The first one consists in determining the location and the shape of the unknown rigid inclusion $\mathscr{B}^{*}$ using the KohnVogelius formulation. The idea is to minimize a misfit functional between the solutions of two forward problems. One of them is associated to the Neumann data $\varphi$ whereas the other one contains information on the Dirichlet data $\mathscr{U}_{d}$. In the second part, to minimize this misfit functional, we resort to the topological sensitivity analysis.

\subsection{The Kohn-Vogelius formulation}

The Kohn-Vogelius formulation is a self regularization technique and reformulate the inverse problem into a shape optimization one. For an admissible rigid inclusion $\mathscr{B} \in \mathrm{V}_{\text {ad }}$; the Kohn-Vogelius formulation consists in splitting the over-determined boundary value problem 2.2 in two auxiliary problems. The first one is called the Neumann problem and associated to the traction field $\varphi$ :

$\left(\mathscr{P}_{N}\right) \quad$ Find $\psi^{N} \in H^{1}(\Omega \backslash \overline{\mathscr{B}})$ solving $\begin{cases}-\operatorname{div} \sigma\left(\psi^{N}\right)=0 & \text { in } \Omega \backslash \overline{\mathscr{B}}, \\ \sigma\left(\psi^{N}\right) \mathbf{n}=\varphi & \text { on } \partial \Omega, \\ \psi^{N}=0 & \text { on } \partial \mathscr{B} .\end{cases}$

The second one is associated to the measured displacement $\mathscr{U}_{d}$ :

$\left(\mathscr{P}_{D}\right) \quad$ Find $\psi^{D} \in H^{1}(\Omega \backslash \overline{\mathscr{B}})$ solving $\begin{cases}-\operatorname{div} \sigma\left(\psi^{D}\right)=0 & \text { in } \Omega \backslash \overline{\mathscr{B}}, \\ \psi^{D}=\mathscr{U}_{d} & \text { on } \partial \Omega, \\ \psi^{D}=0 & \text { on } \partial \mathscr{B} .\end{cases}$

For some results concerning the existence and uniqueness of solutions of problems $\mathscr{P}_{N}$ and $\mathscr{P}_{D}$, we refer to 16$]$.

We remark here that if the admissible rigid inclusion $\mathscr{B}$ coincides with the actual one $\mathscr{B}^{*}$ then the misfit between the solutions vanishes $\psi^{N}=\psi^{D}$ in $\Omega \backslash \overline{\mathscr{B}}{ }^{*}$. According to this observation, the given inverse problem can be formulated as a shape optimization one where the unknown inclusion $\mathscr{B}^{*}$ can be characterized as a solution to the following shape optimization problem:

$\left(\mathscr{P}_{\text {op }}\right) \quad$ Find $\mathscr{B}^{*} \subset \Omega$ such that $\mathscr{K}\left(\Omega \backslash \overline{\mathscr{B}^{*}}\right)=\min _{\mathscr{B} \in \mathrm{V}_{\text {ad }}} \mathscr{K}(\Omega \backslash \overline{\mathscr{B}})$,

where $\mathscr{K}$ is a shape function defined as

$$
\mathscr{K}(\Omega \backslash \overline{\mathscr{B}})=\mathscr{J}\left(\psi^{N}, \psi^{D}\right)
$$


with $\mathscr{J}$ a given cost functional defined on $H^{1}(\Omega \backslash \overline{\mathscr{B}})$, measuring the difference between the Dirichlet and Neumann problem solutions.

In this work, we will use the following Kohn-Vogelius type cost functional,

$$
\mathscr{J}\left(\psi^{N}, \psi^{D}\right)=\int_{\Omega \backslash \mathscr{B}} \sigma\left(\psi^{N}-\psi^{D}\right): e\left(\psi^{N}-\psi^{D}\right) \mathrm{d} x, \quad \forall \mathscr{B} \in \mathrm{V}_{\mathrm{ad}}
$$

This function is positive and vanishes only if $\psi^{N}=\psi^{D}$, which is the case when the parameter $\mathscr{B}$ fits the inclusion. We can notice that, integrating by parts the expression of $\mathscr{J}\left(\psi^{N}, \psi^{D}\right)$, we have:

$$
\mathscr{J}\left(\psi^{N}, \psi^{D}\right)=\int_{\partial \Omega}\left(\psi^{N}-\mathscr{U}_{d}\right) \cdot\left(\varphi-\sigma\left(\psi^{D}\right) \mathbf{n}\right) \mathrm{d} s
$$

The above equality give a boundary expression of $\mathscr{K}$.

This kind of cost function was originally introduced by Wexler et al. in 57 proposed a procedure to reconstruct the unknown impedance from the knowledge of solution on the boundary of the domain. Then Khon and Vogelius in [42] suggested a modification of Wexler's procedure to make it an alternating direction one by proposing a new misfit cost functional. Since then, this formulation has been proved to be extremely useful in the treatment of various inverse problems $13,18,22,28,35$, and so on.

Regarding the minimizer of $\mathscr{P}$ op , we now show the following uniqueness result.

Proposition 3.1. Let $\left(\varphi, \mathscr{U}_{d}\right) \in H^{-1 / 2}(\partial \Omega) \times H^{1 / 2}(\partial \Omega)$ be given nontrivial Cauchy data. We assume that there exists $\mathscr{B}^{*} \in \mathrm{V}_{\text {ad }}$ such that the problem 2.2 has a solution then $\mathscr{B}^{*}$ is the unique minimum of the shape functional $\mathscr{K}$ :

$$
\mathscr{B}^{*}=\underset{\mathscr{B} \in \mathrm{V}_{\text {ad }}}{\arg \min } \mathscr{K}(\Omega \backslash \overline{\mathscr{B}})
$$

Proof. If $\mathscr{B}^{*} \in \mathrm{V}_{\mathrm{ad}}$ is the solution of the inverse problem 2.2$)$, then $\psi^{N}\left(\mathscr{B}^{*}\right)=\psi^{D}\left(\mathscr{B}^{*}\right)$ and $\mathscr{B}^{*}$ is the minimum of the functional $\mathscr{K}$ with $\mathscr{K}\left(\Omega \backslash \overline{\mathscr{B}^{*}}\right)=0$. Let $\mathscr{B} \in \mathrm{V}_{\text {ad }}$ be another minimizer of $\mathscr{K}$. Then $\psi^{N}(\mathscr{B})$ verifies the problem 2.1 with $\psi^{N}(\mathscr{B})=\mathscr{U}_{d}$ on $\partial \Omega$. By using the uniqueness Theorem 2.1, we obtain $\mathscr{B}=\mathscr{B}^{*}$.

Next, to solve the shape optimization problem $\left(\mathscr{P}_{\mathrm{op}}\right)$, we introduce the topological sensitivity analysis method.

\subsection{Topological sensitivity analysis}

The main idea of the topological sensitivity consists in studying the variation of a shape functional with respect to an infinitesimal singular domain perturbation, such as the insertion of cavities, inclusions, source-terms or even cracks $[5,6,12,14,17,19,23,24,34$. 
Let us briefly browse the history of this method. Its main idea was first introduced by Schumacher [54] in the context of compliance minimization. In the same context, Sokolowski and Zochowski 56 gave some mathematical justifications in the plane stress case and generalized it to various cost functions. A topological sensitivity framework using an adaptation of the adjoint method and truncation technique was then introduced by Masmoudi [45] for the Laplace equation. It was generalized in 29] to the elasticity equations in the case of arbitrarily shaped holes. By using the adjoint method and the domain truncation technique, the topological gradient has been calculated for several equations such as Laplace [31], Stokes [32, quasi-Stokes [33], Navier-Stokes [11], elastodynamic [27], Maxwell [46] and Helmholtz [53] and many other equations.

To present the idea of this method, let us consider a perturbation of $\Omega$ confined in a small region $\mathscr{B}_{z, \varepsilon}$ of size $\varepsilon$ and has the form $\mathscr{B}_{z, \varepsilon}=z+\varepsilon \mathscr{C}$, where $z \in \Omega$ and $\mathscr{C} \subset \mathbb{R}^{2}$ is a fixed open and bounded set containing the origin, whose boundary $\partial \mathscr{C}$ is connected and piecewise of class $C^{1}$. Then the topological sensitivity analysis leads to an asymptotic expansion of the shape functional $\mathscr{K}$ on the form:

$$
\mathscr{K}\left(\Omega \backslash \overline{\mathscr{B}_{z, \varepsilon}}\right)=\mathscr{K}(\Omega)+\xi(\varepsilon) \delta \mathscr{K}(z)+o(\xi(\varepsilon)), \quad \forall z \in \Omega
$$

where

- $\varepsilon \mapsto \xi(\varepsilon)$ is a scalar positive function verifying $\lim _{\varepsilon \rightarrow 0} \xi(\varepsilon)=0$ and describes the behavior of the variation $\mathscr{K}(\Omega \backslash \overline{\mathscr{B}} z, \varepsilon)-\mathscr{K}(\Omega)$ with respect to $\varepsilon$.

- The function $z \mapsto \delta \mathscr{K}(z)$ measures the sensitivity of the shape functional $\mathscr{K}$ with respect to a geometry perturbation around the point $z$. The function $\delta \mathscr{K}$ is called the "topological gradient". Mathematically, $\delta \mathscr{K}$ can be expressed as

$$
\delta \mathscr{K}(z):=\lim _{\varepsilon \rightarrow 0} \frac{\mathscr{K}(\Omega \backslash \overline{\mathscr{B}} z, \varepsilon)-\mathscr{K}(\Omega)}{\xi(\varepsilon)} .
$$

- The shape functional $\mathscr{K}$ associated with the topologically perturbed domain $\Omega \backslash \overline{\mathscr{B}} z, \varepsilon$ is written as

$$
\mathscr{K}\left(\Omega \backslash \overline{\mathscr{B}_{z, \varepsilon}}\right)=\int_{\Omega \backslash \overline{\mathscr{B}_{z, \varepsilon}}} \sigma\left(\psi_{\varepsilon}^{D}-\psi_{\varepsilon}^{N}\right): e\left(\psi_{\varepsilon}^{D}-\psi_{\varepsilon}^{N}\right) \mathrm{d} x,
$$

with $\psi_{\varepsilon}^{N}$ and $\psi_{\varepsilon}^{D}$ solve respectively problems $\left(\mathscr{P}_{N}^{\varepsilon}\right)$ and $\mathscr{P}_{D}^{\varepsilon}$.

$\left(\mathscr{P}_{N}^{\varepsilon}\right) \quad$ Find $\psi_{\varepsilon}^{N} \in H^{1}\left(\Omega \backslash \overline{\mathscr{B}_{z, \varepsilon}}\right)$ solving $\begin{cases}-\operatorname{div} \sigma\left(\psi_{\varepsilon}^{N}\right)=0 & \text { in } \Omega \backslash \overline{\mathscr{B}_{z, \varepsilon}}, \\ \sigma\left(\psi_{\varepsilon}^{N}\right) \mathbf{n}=\varphi & \text { on } \partial \Omega, \\ \psi_{\varepsilon}^{N}=0 & \text { on } \partial \mathscr{B}_{z, \varepsilon}\end{cases}$ 
and

$$
\text { Find } \psi_{\varepsilon}^{D} \in H^{1}\left(\Omega \backslash \overline{\mathscr{B}_{z, \varepsilon}}\right) \text { solving } \begin{cases}-\operatorname{div} \sigma\left(\psi_{\varepsilon}^{D}\right)=0 & \text { in } \Omega \backslash \overline{\mathscr{B}_{z, \varepsilon}} \\ \psi_{\varepsilon}^{D}=\mathscr{U}_{d} & \text { on } \partial \Omega \\ \psi_{\varepsilon}^{D}=0 & \text { on } \partial \mathscr{B}_{z, \varepsilon}\end{cases}
$$

In the particular case, if $\varepsilon=0$, we will consider as a convention that $\mathscr{B}_{z, 0}=\emptyset$, and consequently $\Omega \backslash \overline{\mathscr{B}_{z, 0}}=\Omega$. Therefore, we denote by $\psi_{0}^{N}$ and $\psi_{0}^{D}$ respectively the solutions to the following unperturbed problems:

$$
\begin{aligned}
& \left(\mathscr{P}_{N}^{0}\right) \quad \text { Find } \psi_{0}^{N} \in H^{1}(\Omega) \text { solving } \begin{cases}-\operatorname{div} \sigma\left(\psi_{0}^{N}\right)=0 & \text { in } \Omega, \\
\sigma\left(\psi_{0}^{N}\right) \mathbf{n}=\varphi & \text { on } \partial \Omega,\end{cases} \\
& \left(\mathscr{P}_{D}^{0}\right) \\
& \text { Find } \psi_{0}^{D} \in H^{1}(\Omega) \text { solving } \begin{cases}-\operatorname{div} \sigma\left(\psi_{0}^{D}\right)=0 & \text { in } \Omega, \\
\psi_{0}^{D}=\mathscr{U}_{d} & \text { on } \partial \Omega .\end{cases}
\end{aligned}
$$

Then the shape functional $\mathscr{K}$ associated to the unperturbed domain $\Omega$ is written as

$$
\mathscr{K}(\Omega)=\int_{\Omega} \sigma\left(\psi_{0}^{N}-\psi_{0}^{D}\right): e\left(\psi_{0}^{N}-\psi_{0}^{D}\right) \mathrm{d} x .
$$

In order to minimize the shape functional $\mathscr{K}$, the best location to insert a small inclusion $\mathscr{B}_{z, \varepsilon}$ in $\Omega$ is where $\delta \mathscr{K}$ is the most negative. In fact, if $\delta \mathscr{K}(z)<0$ we have $\mathscr{K}\left(\Omega \backslash \overline{\mathscr{B}_{z, \varepsilon}}\right) \leq \mathscr{K}(\Omega)$ for small $\varepsilon>0$. Particularly, the solution of the optimization problem

$$
\min _{\mathscr{B}_{z, \varepsilon} \in \mathrm{V}_{\mathrm{ad}}} \mathscr{K}(\Omega \backslash \overline{\mathscr{B}} z, \varepsilon)
$$

is given by $\mathscr{B}_{z^{*}, \varepsilon}^{*}=z^{*}+\varepsilon \mathscr{C}$ where $z^{*} \in \Omega$ such that $\delta \mathscr{K}\left(z^{*}\right)<0$ and $\delta \mathscr{K}\left(z^{*}\right)<\delta \mathscr{K}(z)$, $\forall z \in \Omega$.

Remark 3.2. Notice that the solution $\psi_{0}^{N}$ of the unperturbed Neumann problem $\mathscr{P}_{N}^{0}$ has a unique solution $\psi_{0}^{N}$ up to an additive constant. In order to ensure uniqueness we add the following normalization condition:

$$
\int_{\partial \Omega} \varphi \cdot w \mathrm{~d} s=0, \quad \forall w \in \mathscr{V},
$$

where $\mathscr{V}$ denotes the rigid motions space of the domain $\Omega$ defined as

$$
\mathscr{V}=\left\{v \in H^{1}(\Omega), e(v)=0\right\} .
$$

In practice, to avoid the normalization condition $(3.2)$, one can solve the following variational problem:

$$
\int_{\Omega} \sigma\left(\psi_{0}^{N}\right): e(w) \mathrm{d} x+\int_{\Omega} \varsigma \psi_{0}^{N} \cdot w \mathrm{~d} x=\int_{\partial \Omega} \varphi \cdot w \mathrm{~d} s, \quad \forall w \in \mathrm{H}^{1}(\Omega)
$$

where $\varsigma$ is a small parameter $\left(\sim 10^{-10}\right)$. 
Remark 3.3. For the sake of simplicity in what follows we will work with an origin-centered inclusion (i.e., $z=0$ ). The general case comes from a linear change of coordinates. Then, we denote by $\mathscr{B}_{z, \varepsilon}=\mathscr{B}_{0, \varepsilon}=: \mathscr{B}_{\varepsilon}$ also consider $\Omega_{\varepsilon}:=\Omega \backslash \overline{\mathscr{B}_{0, \varepsilon}}$.

In the next section, we present the theoretical aspect of the proposed method. We derive a topological sensitivity analysis for the linear elasticity operator. The obtained results are valid for a Dirichlet and/or Neumann boundary condition on $\partial \Omega$ (see Proposition 4.10). In Section 4.2, from the obtained results, we will deduce an asymptotic expansion for the Kohn-Vogelius functional $\mathscr{K}$ (see Theorem 4.13).

\section{Asymptotic analysis}

\subsection{General case}

This section is devoted to the topological sensitivity analysis for the linear elasticity operator with respect to the presence of a small inclusion $\mathscr{B}_{\varepsilon}=\varepsilon \mathscr{C}$ inside the background domain $\Omega$. This question has been investigated in the past by Garreau et al. [29] and Giusti et al. [30]. In the first reference they considered a Neumann boundary condition on the small inclusion obtaining general results in two and three dimensional cases. They established a topological sensitivity analysis based on the truncation technique and the sensitivity analysis of the Dirichlet-to-Neumann operator. In the work of Giusti et al. they obtained a topological derivative of inclusion in two dimensions in linear elasticity problem using the Topological-Shape Sensitivity Method.

Here, we do not use the truncation technique and the Topological-Shape Sensitivity Method, but we follow the simplified approach presented in [1] (see also [2,25]). However, contrary to the problem studied in [29], we impose Dirichlet boundary conditions on the interior boundary of $\mathscr{B}_{\varepsilon}$. We propose a simple and rigorous mathematical analysis based on a preliminary estimate describing the influence of the geometric perturbation $\mathscr{B}_{\varepsilon}$ on the elasticity problem solution. In order to derive a topological sensitivity analysis valid for the problems $\left(\mathscr{P}_{N}^{\varepsilon}\right)$ and $\left(\mathscr{P}_{D}^{\varepsilon}\right)$, we introduce the following auxiliary problem:

$$
\begin{cases}-\operatorname{div} \sigma\left(\psi_{\varepsilon}\right)=0 & \text { in } \Omega_{\varepsilon}, \\ \sigma\left(\psi_{\varepsilon}\right) \mathbf{n}=\varphi_{n} & \text { on } \Sigma, \\ \psi_{\varepsilon}=0 & \text { on } \Gamma, \\ \psi_{\varepsilon}=0 & \text { on } \partial \mathscr{B}_{\varepsilon},\end{cases}
$$

where $\varphi_{n} \in H^{-1 / 2}(\Sigma)$ is a given data, with $\Sigma$ and $\Gamma$ form a non-overlapping decomposition of the boundary $\partial \Omega$ : $\Sigma$ and $\Gamma$ are relatively open, $\partial \Omega=\bar{\Sigma} \cup \bar{\Gamma}$, and $\Sigma \cap \Gamma=\emptyset$. 
In the absence of a rigid inclusion (i.e., $\varepsilon=0) \Omega_{0}=\Omega$ and $\psi_{0}$ solves

$$
\begin{cases}-\operatorname{div} \sigma\left(\psi_{0}\right)=0 & \text { in } \Omega, \\ \sigma\left(\psi_{0}\right) \mathbf{n}=\varphi_{n} & \text { on } \Sigma, \\ \psi_{0}=0 & \text { on } \Gamma .\end{cases}
$$

Remark 4.1. In the particular case $\Sigma=\partial \Omega(\Gamma=\emptyset)$, the problem 4.2 has a unique solution $\psi_{0}$, up to an additive constant. To ensure uniqueness, the boundary force $\varphi_{n}$ should satisfy the compatibility condition:

$$
\int_{\Sigma} \varphi_{n} \cdot w \mathrm{~d} s=0, \quad \forall w \in \mathscr{V}
$$

Consider now the shape function

$$
\mathscr{J}\left(\Omega_{\varepsilon}\right)=\int_{\Omega_{\varepsilon}} \sigma\left(\psi_{\varepsilon}\right): e\left(\psi_{\varepsilon}\right) \mathrm{d} x
$$

The aim is to derive an asymptotic expansion of the shape function $\mathscr{J}$ with respect to the presence of a small rigid inclusion $\mathscr{B}_{\varepsilon}$ in $\Omega$. More precisely, we study the variation $\mathscr{J}\left(\Omega_{\varepsilon}\right)-\mathscr{J}(\Omega)$ with respect to $\varepsilon$ and establish an asymptotic formula of the form

$$
\mathscr{J}\left(\Omega_{\varepsilon}\right)-\mathscr{J}(\Omega)=\xi(\varepsilon) \delta \mathscr{J}(0)+o(\xi(\varepsilon)) .
$$

We recall that we will determine the above asymptotic expansion of $\mathscr{J}$ only for the case of an origin-centered inclusion (see Remark 3.3. .

The variation of the shape function $\mathscr{J}$ reads

$$
\begin{aligned}
\mathscr{J}\left(\Omega_{\varepsilon}\right)-\mathscr{J}(\Omega)= & \int_{\Omega_{\varepsilon}} \sigma\left(\psi_{\varepsilon}\right): e\left(\psi_{\varepsilon}\right) \mathrm{d} x-\int_{\Omega} \sigma\left(\psi_{0}\right): e\left(\psi_{0}\right) \mathrm{d} x \\
= & \int_{\mathscr{B}_{\varepsilon}} \sigma\left(\psi_{0}\right): e\left(\psi_{0}\right) \mathrm{d} x+\int_{\Omega_{\varepsilon}} \sigma\left(\psi_{\varepsilon}-\psi_{0}\right): e\left(\psi_{\varepsilon}-\psi_{0}\right) \mathrm{d} x \\
& +2 \int_{\Omega} \sigma\left(\psi_{0}\right): e\left(\psi_{\varepsilon}-\psi_{0}\right) \mathrm{d} x .
\end{aligned}
$$

Remark 4.2. In the equality (4.3), the solution $\psi_{\varepsilon}$ of problem 4.1 is extended by zero inside the region $\mathscr{B}_{\varepsilon}$. Its extension will be denoted by $\psi_{\varepsilon}$ throughout the rest of the paper.

Let us introduce an adjoint state $\vartheta_{0}$ solution to the following auxiliary variational problem

Find $\vartheta_{0} \in H_{\Gamma}^{1}(\Omega)$, such that

$$
\int_{\Omega} \sigma(w): e\left(\vartheta_{0}\right) \mathrm{d} x=-2 \int_{\Omega} \sigma\left(\psi_{0}\right): e(w) \mathrm{d} x, \quad \forall w \in H_{\Gamma}^{1}(\Omega) .
$$


The space $H_{\Gamma}^{1}(\Omega)$ is defined as

$$
H_{\Gamma}^{1}(\Omega)=\left\{w \in H^{1}(\Omega) ; w=0 \text { on } \Gamma\right\}
$$

Then $\vartheta_{0}$ it can be interpreted as the solution to the following adjoint problem

$$
\begin{cases}-\operatorname{div} \sigma\left(\vartheta_{0}\right)=-D \mathrm{~J}_{0}\left(\psi_{0}\right) & \text { in } \Omega, \\ \sigma\left(\vartheta_{0}\right) \mathbf{n}=0 & \text { on } \Sigma, \\ \vartheta_{0}=0 & \text { on } \Gamma,\end{cases}
$$

associated to the minimization problem

$$
\min _{w \in H_{\Gamma}^{1}(\Omega)} \mathrm{J}_{0}(w)
$$

with $\mathrm{J}_{0}$ being the cost functional defined by

$$
\mathrm{J}_{0}(w)=\int_{\Omega} \sigma(w): e(w) \mathrm{d} x, \quad \forall w \in H_{\Gamma}^{1}(\Omega) .
$$

By taking $w=\psi_{\varepsilon}-\psi_{0}$ as a test function in (4.4) and using Remark 4.2, we deduce that the last term of 4.3 can be rewritten as

$$
\begin{aligned}
2 \int_{\Omega} \sigma\left(\psi_{0}\right): e\left(\psi_{\varepsilon}-\psi_{0}\right) \mathrm{d} x & =-\int_{\Omega} \sigma\left(\psi_{\varepsilon}-\psi_{0}\right): e\left(\vartheta_{0}\right) \mathrm{d} x \\
& =\int_{\mathscr{B}_{\varepsilon}} \sigma\left(\psi_{0}\right): e\left(\vartheta_{0}\right) \mathrm{d} x+\int_{\Omega_{\varepsilon}} \sigma\left(\psi_{0}-\psi_{\varepsilon}\right): e\left(\vartheta_{0}\right) \mathrm{d} x
\end{aligned}
$$

Consequently, the variation $\mathscr{J}\left(\Omega_{\varepsilon}\right)-\mathscr{J}(\Omega)$ can be decomposed as:

$$
\begin{aligned}
\mathscr{J}\left(\Omega_{\varepsilon}\right)-\mathscr{J}(\Omega)= & \int_{\mathscr{B}_{\varepsilon}} \sigma\left(\psi_{0}\right): e\left(\psi_{0}\right) \mathrm{d} x+\int_{\mathscr{B}_{\varepsilon}} \sigma\left(\psi_{0}\right): e\left(\vartheta_{0}\right) \mathrm{d} x \\
& +\int_{\Omega_{\varepsilon}} \sigma\left(\psi_{0}-\psi_{\varepsilon}\right): e\left(\vartheta_{0}\right) \mathrm{d} x+\int_{\Omega_{\varepsilon}} \sigma\left(\psi_{\varepsilon}-\psi_{0}\right): e\left(\psi_{\varepsilon}-\psi_{0}\right) \mathrm{d} x .
\end{aligned}
$$

In the next section, we will derive an estimate for each term on the right-hand side of (4.5).

\subsubsection{Preliminary estimates}

The following lemma gives an estimate for the first term of 4.5 .

Lemma 4.3. We have

$$
\int_{\mathscr{B}_{\varepsilon}} \sigma\left(\psi_{0}\right): e\left(\psi_{0}\right) \mathrm{d} x=O\left(\varepsilon^{2}\right)
$$


Proof. We can write

$$
\begin{aligned}
\int_{\mathscr{B}_{\varepsilon}} \sigma\left(\psi_{0}\right): e\left(\psi_{0}\right) \mathrm{d} x= & \varepsilon^{2}|\mathcal{S}| \sigma\left(\psi_{0}\right)(0): e\left(\psi_{0}\right)(0) \\
& +\int_{\mathscr{B}_{\varepsilon}}\left\{\sigma\left(\psi_{0}\right): e\left(\psi_{0}\right)-\sigma\left(\psi_{0}\right)(0): e\left(\psi_{0}\right)(0)\right\} \mathrm{d} x .
\end{aligned}
$$

We obtain by a change of variable $x=\varepsilon y$ that

$$
\begin{aligned}
& \int_{\mathscr{B}_{\varepsilon}}\left\{\sigma\left(\psi_{0}\right): e\left(\psi_{0}\right)-\sigma\left(\psi_{0}\right)(0): e\left(\psi_{0}\right)(0)\right\} \mathrm{d} x \\
= & \varepsilon^{2} \int_{\mathscr{C}}\left\{\sigma\left(\psi_{0}\right)(\varepsilon y): e\left(\psi_{0}\right)(\varepsilon y)-\sigma\left(\psi_{0}\right)(0): e\left(\psi_{0}\right)(0)\right\} \mathrm{d} y .
\end{aligned}
$$

Since $\psi_{0}$ is of $C^{1}$ in a neighborhood of the origin, we obtain immediately with the help of a Taylor expansion that

$$
\int_{\mathscr{C}}\left\{\sigma\left(\psi_{0}\right)(\varepsilon y): e\left(\psi_{0}\right)(\varepsilon y)-\sigma\left(\psi_{0}\right)(0): e\left(\psi_{0}\right)(0)\right\} \mathrm{d} y=O(\varepsilon)
$$

Hence,

$$
\int_{\mathscr{B}_{\varepsilon}} \sigma\left(\psi_{0}\right): e\left(\psi_{0}\right) \mathrm{d} x=O\left(\varepsilon^{2}\right)
$$

In a similar manner, we derive the following lemma. It describes an estimate of the second term of right-hand side of (4.5).

Lemma 4.4. We have

$$
\int_{\mathscr{B}_{\varepsilon}} \sigma\left(\psi_{0}\right): e\left(\vartheta_{0}\right) \mathrm{d} x=O\left(\varepsilon^{2}\right)
$$

To estimate the third term of 4.5, we will need two preliminary lemmas. The first one concerns an estimate of the norm $\|\cdot\|_{1 / 2, \partial \mathscr{B}_{\varepsilon}}$ of an uniformly bounded function. In the second lemma, we give an asymptotic expansion of the solution of the perturbed elasticity problem 4.1.

Lemma 4.5. Let $\varepsilon \in(0,1 / 2)$. If $\psi \in H^{1}(\Omega)$ is such that is restriction to $\overline{\mathscr{B}_{1}}=\overline{\mathscr{C}}$ is $C^{1}$, then there exists a constant $c>0$ independent of $\varepsilon$ such that

$$
\|\psi\|_{1 / 2, \partial \mathscr{B}_{\varepsilon}} \leq \frac{c}{\sqrt{-\log (\varepsilon)}} .
$$

Proof. Thanks to Theorem 7.3 , there exists a constant $c>0$ (independent of $\varepsilon$ ) such that

$$
\|\psi\|_{1 / 2, \partial \mathscr{B}_{\varepsilon}} \leq \frac{c \varepsilon^{-1 / 2}}{\sqrt{-\log (\varepsilon)}}\|\psi\|_{L^{2}\left(\partial \mathscr{B}_{\varepsilon}\right)}+c\left(\iint_{\partial \mathscr{B}_{\varepsilon} \times \partial \mathscr{B}_{\varepsilon}} \frac{|\psi(x)-\psi(y)|^{2}}{|x-y|^{2}} \mathrm{~d} s(x) \mathrm{d} s(y)\right)^{1 / 2}
$$


Using the changes of variables $x=\varepsilon x_{1}$ and $y=\varepsilon y_{1}$ and Taylor formula, we have

$$
\begin{aligned}
& \psi\left(\varepsilon x_{1}\right)=\psi(0)+\varepsilon \nabla \psi\left(\xi_{x_{1}}\right) x_{1} \quad \text { with } \xi_{x_{1}} \in \mathscr{B}_{\varepsilon}, \\
& \psi\left(\varepsilon y_{1}\right)=\psi(0)+\varepsilon \nabla \psi\left(\xi_{y_{1}}\right) y_{1} \quad \text { with } \xi_{y_{1}} \in \mathscr{B}_{\varepsilon} \text {. }
\end{aligned}
$$

Then there exists a constant $c>0$ independent of $\varepsilon$ such that

$$
\begin{aligned}
& \left(\iint_{\partial \mathscr{B}_{\varepsilon} \times \partial \mathscr{B}_{\varepsilon}} \frac{|\psi(x)-\psi(y)|^{2}}{|x-y|^{2}} \mathrm{~d} s(x) \mathrm{d} s(y)\right)^{1 / 2} \\
= & \left(\iint_{\partial \mathscr{C} \times \partial \mathscr{C}} \varepsilon^{2} \frac{\left|\varepsilon\left(\nabla \psi\left(\xi_{x_{1}}\right) x_{1}-\nabla \psi\left(\xi_{y_{1}}\right) y_{1}\right)\right|^{2}}{\varepsilon^{2}\left|x_{1}-y_{1}\right|^{2}} \mathrm{~d} s(x) \mathrm{d} s(y)\right)^{1 / 2} \leq c \varepsilon .
\end{aligned}
$$

Moreover, using the change of variable $x=\varepsilon y$ and that $\psi$ is uniformly bounded on $\partial \mathscr{B}_{\varepsilon}$, then there exists a constant $c>0$ independent of $\varepsilon$ such that

$$
\|\psi\|_{L^{2}\left(\partial \mathscr{B}_{\varepsilon}\right)} \leq c \varepsilon^{1 / 2}
$$

Consequently, we get

$$
\|\psi\|_{1 / 2, \partial \mathscr{B}_{\varepsilon}} \leq \frac{c \varepsilon^{-1 / 2}}{\sqrt{-\log (\varepsilon)}} \varepsilon^{1 / 2}+c \varepsilon \leq \frac{c}{\sqrt{-\log (\varepsilon)}} .
$$

Before establishing the asymptotic expansion of $\psi_{\varepsilon}$ (solution of problem (4.1)) let us recall the expression of the fundamental solution $G$ to the linear elasticity system in $\mathbb{R}^{2}$

$$
G(x)=\beta \log (\|x\|) \mathrm{I}+\gamma e_{r}^{t} e_{r},
$$

with $e_{r}=x /\|x\|$; that is $-\operatorname{div} \sigma\left(G_{j}\right)=\delta e_{j}$, where $G_{j}$ denotes the $j^{\text {th }}$ column of $G,\left(e_{j}\right)_{j=1}^{2}$ is the canonical basis of $\mathbb{R}^{2}$ and $\delta$ is the Dirac distribution. The constants $\beta$ and $\gamma$ are defined as

$$
\beta=-\frac{\lambda+3 \mu}{4 \pi \mu(\lambda+2 \mu)} \quad \text { and } \quad \gamma=\frac{\lambda+\mu}{4 \pi \mu(\lambda+2 \mu)} \quad \text { (plane strain). }
$$

For plane stress, $\lambda^{*}=2 \mu \lambda /(\lambda+2 \mu)$ must be substituted for $\lambda$.

Lemma 4.6. The solution $\psi_{\varepsilon}$ of problem 4.1) admits the following asymptotic expansion:

$$
\psi_{\varepsilon}(x)=\psi_{0}(x)+\mathrm{T}_{\varepsilon}(\mathscr{H}(x)-\zeta(x))+O_{H^{1}\left(\Omega_{\varepsilon}\right)}\left(\frac{1}{-\log (\varepsilon)}\right),
$$

where $\zeta \in H^{1}(\Omega)$ solves the following linear elasticity problem

$$
\begin{cases}-\operatorname{div} \sigma(\zeta)=0 & \text { in } \Omega \\ \zeta=\mathscr{H} & \text { on } \Gamma\end{cases}
$$


with $\mathrm{T}_{\varepsilon}=\frac{1}{-\log (\varepsilon)}$ and

$$
\mathscr{H}(x)=\frac{-1}{\beta} G(x) \psi_{0}(0) .
$$

The notation $O_{H^{1}\left(\Omega_{\varepsilon}\right)}\left(\frac{1}{-\log (\varepsilon)}\right)$ means that there exists a constant $c>0$ (independent of $\varepsilon)$ and $\varepsilon_{1}>0$ such that for all $0<\varepsilon<\varepsilon_{1}$

$$
\left\|\psi_{\varepsilon}(x)-\psi_{0}(x)-\mathrm{T}_{\varepsilon}(\mathscr{H}(x)-\zeta(x))\right\|_{1, \Omega_{\varepsilon}} \leq \frac{c}{-\log (\varepsilon)} .
$$

To prove Lemma 4.6, we need to establish the following lemma. We postpone its technical proof in Section 7 .

Lemma 4.7. Let $\varepsilon>0$. For $\theta_{N} \in H^{-1 / 2}(\Sigma), \theta_{D} \in H^{1 / 2}(\Gamma)$ and $\theta \in H^{1 / 2}\left(\partial \mathscr{B}_{z, \varepsilon}\right)$, let $v_{\varepsilon} \in H^{1}\left(\Omega \backslash \overline{\mathscr{B}_{z, \varepsilon}}\right)$ be the solution of the linear elasticity problem

$$
\begin{cases}-\operatorname{div} \sigma\left(v_{\varepsilon}\right)=0 & \text { in } \Omega \backslash \overline{\mathscr{B}_{z, \varepsilon}}, \\ \sigma\left(v_{\varepsilon}\right) \mathbf{n}=\theta_{N} & \text { on } \Sigma, \\ v_{\varepsilon}=\theta_{D} & \text { on } \Gamma, \\ v_{\varepsilon}=\theta & \text { on } \partial \mathscr{B}_{z, \varepsilon} .\end{cases}
$$

Then there exists a constant $c>0$ (independent of $\varepsilon$ ) such that

$$
\left\|v_{\varepsilon}\right\|_{1, \Omega \backslash \overline{\mathscr{B}_{z, \varepsilon}}} \leq c\left(\left\|\theta_{N}\right\|_{-1 / 2, \Sigma}+\left\|\theta_{D}\right\|_{1 / 2, \Gamma}+\|\theta\|_{1 / 2, \partial \mathscr{B}_{z, \varepsilon}}\right) .
$$

Now, we turn to prove Lemma 4.6.

Proof of Lemma 4.6. In order to simplify the notations, let us define

$$
r_{\varepsilon}=\psi_{\varepsilon}-\psi_{0}-\mathrm{T}_{\varepsilon}(\mathscr{H}-\zeta)
$$

where $\psi_{\varepsilon}$ and $\psi_{0}$ solve respectively problem 4.1) and (4.2). One can easily remak that $r_{\varepsilon}$ is solution to the system

$$
\begin{cases}-\operatorname{div} \sigma\left(r_{\varepsilon}\right)=0 & \text { in } \Omega_{\varepsilon}, \\ \sigma\left(r_{\varepsilon}\right) \mathbf{n}=\frac{1}{\log (\varepsilon)}(\sigma(\mathscr{H}) \mathbf{n}-\sigma(\zeta) \mathbf{n}) & \text { on } \Sigma, \\ r_{\varepsilon}=\frac{1}{\log (\varepsilon)}(\mathscr{H}-\zeta) & \text { on } \Gamma, \\ r_{\varepsilon}=-\psi_{0}-\mathrm{T}_{\varepsilon}(\mathscr{H}-\zeta) & \text { on } \partial \mathscr{B}_{\varepsilon} .\end{cases}
$$

Thanks to Lemma 4.7 that there exists a constant $c>0$, independent of $\varepsilon$ such that

$$
\begin{aligned}
\left\|r_{\varepsilon}\right\|_{1, \Omega_{\varepsilon}} \leq c & \left\{\frac{1}{-\log (\varepsilon)}\left(\|\sigma(\mathscr{H}) \mathbf{n}\|_{-1 / 2, \Sigma}+\|\sigma(\zeta) \mathbf{n}\|_{-1 / 2, \Sigma}+\|\mathscr{H}\|_{1 / 2, \Gamma}+\|\zeta\|_{1 / 2, \Gamma}\right)\right. \\
& \left.+\left\|\psi_{0}+\mathrm{T}_{\varepsilon}(\mathscr{H}-\zeta)\right\|_{1 / 2, \partial \mathscr{B}_{\varepsilon}}\right\}
\end{aligned}
$$


Next we will derive an estimate for each term on the right-hand side of 4.9).

- Estimate of the imposed boundary data on $\Sigma$. We have

$$
\|\sigma(\mathscr{H}) \mathbf{n}\|_{-1 / 2, \Sigma} \leq c|\mathscr{H}|_{1, \Omega \backslash \mathrm{B}(0,1)} \quad \text { and } \quad\|\sigma(\zeta) \mathbf{n}\|_{-1 / 2, \Sigma} \leq c|\zeta|_{1, \Omega_{\varepsilon}} .
$$

Here and in the sequel, $c>0$ is used to denote any constant (independent of $\varepsilon$ ), that may have different values.

Let us first focus on the first estimate on the right-hand side of 4.10). For all $\vartheta \in$ $H^{1 / 2}(\Sigma)$ and all $\phi \in H^{1}(\Omega \backslash \mathbf{B}(0,1))$, extension of $\vartheta$ such that $\phi_{\mid \Gamma}=0$, we have

$$
\begin{aligned}
\langle\sigma(\mathscr{H}) \mathbf{n}, \vartheta\rangle_{-1 / 2,1 / 2, \Sigma} & =\int_{\Omega \backslash \mathbf{B}(0,1)} \sigma(\mathscr{H}): e(\phi) \mathrm{d} x \\
& =\int_{\Omega \backslash \mathbf{B}(0,1)} 2 \mu e(\mathscr{H}): e(\vartheta)+\lambda \operatorname{div}(\mathscr{H}) \operatorname{div}(\vartheta) \mathrm{d} x \\
& \leq c\|e(\mathscr{H})\|_{0, \Omega \backslash \mathbf{B}(0,1)}\|\phi\|_{1, \Omega \backslash \mathbf{B}(0,1)} \\
& \leq c|\mathscr{H}|_{1, \Omega \backslash \mathbf{B}(0,1)}\|\phi\|_{1, \Omega \backslash \mathbf{B}(0,1)} .
\end{aligned}
$$

We recall that the semi-norm $|\cdot|_{1, \Omega}$ is defined by $(7.1)$ and the dual space $H^{-1 / 2}(\Sigma)$ is equipped with the natural norm

$$
\|w\|_{-1 / 2, \Sigma}=\sup \left\{\langle w, v\rangle_{-1 / 2,1 / 2, \Sigma} ; v \in H^{1 / 2}(\Sigma),\|v\|_{1 / 2, \Sigma}=1\right\}
$$

Hence, choosing $\phi$ such that $\|\phi\|_{1, \Omega \backslash \mathbf{B}(0,1)}=\|\vartheta\|_{1 / 2, \Sigma}$, we get

$$
\|\sigma(\mathscr{H}) \mathbf{n}\|_{-1 / 2, \Sigma} \leq c|\mathscr{H}|_{1, \Omega \backslash \mathbf{B}(0,1)} .
$$

For the second estimate in 4.10 we adopt the same analysis just by taking $\Omega_{\varepsilon}$, instead of $\Omega \backslash \mathbf{B}(0,1)$ that we will use.

Now we need an estimate for the functions $\mathscr{H}$ and $\zeta$. For $\mathscr{H}$ we will need a bound for the term $|\mathscr{H}|_{1, \Omega \backslash \mathrm{B}(0,1)}$. To this end, notice that $|\nabla \mathscr{H}|=O(1 /\|x\|)$ and let $R>0$ such that the domain $\Omega \subset \mathrm{B}(0, R)$, then

$$
\begin{aligned}
|\mathscr{H}|_{1, \Omega \backslash \mathbf{B}(0,1)} & \leq|\mathscr{H}|_{1, \mathbf{B}(0, R) \backslash \mathbf{B}(0,1)} \\
& \leq c\left(\int_{\mathbf{B}(0, R) \backslash \mathbf{B}(0,1)} \frac{1}{\|x\|^{2}} \mathrm{~d} x\right)^{1 / 2}=c(2 \pi \log (R))^{1 / 2} .
\end{aligned}
$$

For $\zeta$, from 4.8 , we have

$$
\|\zeta\|_{1, \Omega} \leq c\|\mathscr{H}\|_{1 / 2, \partial \Omega} .
$$

Let $r>0$ be such that the closed ball $\overline{\mathrm{B}(0, r)}$ is included in $\Omega$ and $\mathscr{B}_{\varepsilon} \subset \mathrm{B}(0, r)$. By trace theorem we deduce that

$$
\|\zeta\|_{1, \Omega} \leq c\|\mathscr{H}\|_{1, \Omega \backslash \overline{\mathrm{B}(0, r)}}
$$


Since $\mathscr{H}(x)=\frac{-1}{\beta} G(x) \psi_{0}(0)$ which is bounded if $x$ is away from zero. Then

$$
\|\zeta\|_{1, \Omega} \leq c
$$

Consequently, from 4.10,

$$
\|\sigma(\mathscr{H}) \mathbf{n}\|_{-1 / 2, \Sigma} \leq c \quad \text { and } \quad\|\sigma(\zeta) \mathbf{n}\|_{-1 / 2, \Sigma} \leq c .
$$

Using the same argument as the one used in the deduction of 4.12 , we obtain

$$
\begin{aligned}
\|\mathscr{H}\|_{1 / 2, \Gamma} & \leq c \\
\|\zeta\|_{1 / 2, \Gamma} & \leq c .
\end{aligned}
$$

- Estimate of the imposed boundary data on $\partial \mathscr{B}_{\varepsilon}$. Using the change of variable $x=\varepsilon y$, we have

$$
\begin{aligned}
& \psi_{0}(x)+\mathrm{T}_{\varepsilon}(\mathscr{H}(x)-\zeta(x)) \\
= & \psi_{0}(\varepsilon y)+\frac{1}{-\log (\varepsilon)}\left[\left(\log (\varepsilon\|y\|) \mathrm{I}-\frac{\gamma}{\beta} e_{r}^{t} e_{r}\right) \cdot \psi_{0}(0)-\zeta(\varepsilon y)\right] \\
= & \psi_{0}(\varepsilon y)-\psi_{0}(0)+\frac{1}{-\log (\varepsilon)}\left[\left(\log (\|y\|) \mathrm{I}-\frac{\gamma}{\beta} e_{r}{ }^{t} e_{r}\right) \cdot \psi_{0}(0)-\zeta(\varepsilon y)\right] .
\end{aligned}
$$

Using a Taylor expansion, we obtain $\psi_{0}(\varepsilon y)=\psi_{0}(0)+\varepsilon \nabla \psi_{0}\left(\varsigma_{y}\right) y$ with $\varsigma_{y} \in \mathscr{B}_{\varepsilon}$. Then

$$
\begin{aligned}
& \psi_{0}(x)+\mathrm{T}_{\varepsilon}(\mathscr{H}(x)-\zeta(x)) \\
= & \varepsilon \nabla \psi_{0}\left(\varsigma_{y}\right)+\frac{1}{-\log (\varepsilon)}\left[\left(\log (\|y\|)-\frac{\gamma}{\beta} e_{r}{ }^{t} e_{r}\right) \cdot \psi_{0}(0)-\zeta(\varepsilon y)\right] .
\end{aligned}
$$

Since $\nabla \psi_{0}$ is uniformly bounded on $\mathscr{B}_{\varepsilon}$ and from the boundness of $\zeta$, we get that

$$
\left\|\psi_{0}+\mathrm{T}_{\varepsilon}(\mathscr{H}-\zeta)\right\|_{1 / 2, \partial \mathscr{B}_{\varepsilon}} \leq c_{1} \varepsilon+\frac{c_{2}}{-\log (\varepsilon)} \leq \frac{c}{-\log (\varepsilon)} .
$$

Gathering (4.9), 4.13), 4.14) and 4.15), we have

$$
\left\|\psi_{\varepsilon}-\psi_{0}-\mathrm{T}_{\varepsilon}(\mathscr{H}-\zeta)\right\|_{1, \Omega_{\varepsilon}} \leq \frac{c}{-\log (\varepsilon)} .
$$

Resorting to Lemmas 4.5 and 4.6 , we deduce the following estimate of the third term of 4.5 .

Lemma 4.8. We have

$$
\int_{\partial \mathscr{B}_{\varepsilon}} \sigma\left(\psi_{0}-\psi_{\varepsilon}\right) \mathbf{n} \cdot \vartheta_{0} \mathrm{~d} s=\frac{1}{-\log (\varepsilon)} \frac{4 \pi \mu(\mu+\eta)}{2 \mu+\eta} \psi_{0}(0) \cdot \vartheta_{0}(0)+o\left(\frac{1}{-\log (\varepsilon)}\right),
$$

where the constant $\eta$ is defined by

$$
\eta= \begin{cases}\frac{\mu(3 \lambda+2 \mu)}{\lambda+\mu} & \text { plane stress } \\ \lambda+\mu & \text { plane strain. }\end{cases}
$$


Proof. We have

$$
\begin{aligned}
& \int_{\partial \mathscr{B}_{\varepsilon}}\left(\sigma\left(\psi_{0}-\psi_{\varepsilon}\right) \mathbf{n}\right) \cdot \vartheta_{0} \mathrm{~d} s \\
= & \int_{\partial \mathscr{B}_{\varepsilon}}\left(\sigma\left(\psi_{0}-\psi_{\varepsilon}+\mathrm{T}_{\varepsilon}(\mathscr{H}-\zeta)\right) \mathbf{n}\right) \cdot \vartheta_{0} \mathrm{~d} s-\mathrm{T}_{\varepsilon} \int_{\partial \mathscr{B}_{\varepsilon}}(\sigma(\mathscr{H}-\zeta) \mathbf{n}) \cdot \vartheta_{0} \mathrm{~d} s .
\end{aligned}
$$

Let us first focus on the estimate of the first term on the right-hand side of (4.17). We have

$\left|\int_{\partial \mathscr{B}_{\varepsilon}}\left(\sigma\left(\psi_{0}-\psi_{\varepsilon}+\mathrm{T}_{\varepsilon}(\mathscr{H}-\zeta)\right) \mathbf{n}\right) \cdot \vartheta_{0} \mathrm{~d} s\right| \leq\left\|\sigma\left(\psi_{0}-\psi_{\varepsilon}+\mathrm{T}_{\varepsilon}(\mathscr{H}-\zeta)\right) \mathbf{n}\right\|_{-1 / 2, \partial \mathscr{B}_{\varepsilon}}\left\|\vartheta_{0}\right\|_{1 / 2, \partial \mathscr{B}_{\varepsilon}}$.

Using the same argument as the one used in the deduction of 4.10 , we get

$$
\left\|\sigma\left(\psi_{0}-\psi_{\varepsilon}+\mathrm{T}_{\varepsilon}(\mathscr{H}-\zeta)\right) \mathbf{n}\right\|_{-1 / 2, \partial \mathscr{B}_{\varepsilon}} \leq c\left\|\psi_{0}-\psi_{\varepsilon}+\mathrm{T}_{\varepsilon}(\mathscr{H}-\zeta)\right\|_{1, \Omega_{\varepsilon}}
$$

Thanks to Lemma 4.6, one obtains

$$
\left\|\sigma\left(\psi_{0}-\psi_{\varepsilon}+\mathrm{T}_{\varepsilon}(\mathscr{H}-\zeta)\right) \mathbf{n}\right\|_{-1 / 2, \partial \mathscr{B}_{\varepsilon}} \leq \frac{c}{-\log (\varepsilon)}
$$

Thus, from Lemma 4.5, we deduce that

$$
\begin{aligned}
\left|\int_{\partial \mathscr{B}_{\varepsilon}}\left(\sigma\left(\psi_{0}-\psi_{\varepsilon}+\mathrm{T}_{\varepsilon}(\mathscr{H}-\zeta)\right) \mathbf{n}\right) \cdot \vartheta_{0} \mathrm{~d} s\right| & \leq \frac{c}{-\log (\varepsilon)}\left\|\vartheta_{0}\right\|_{1 / 2, \partial \mathscr{B}_{\varepsilon}} \\
& \leq \frac{c}{(-\log (\varepsilon))^{3 / 2}} .
\end{aligned}
$$

Now, we estimate the last term on the right-hand side of 4.17). We have

$$
\int_{\partial \mathscr{B}_{\varepsilon}}(\sigma(\mathscr{H}-\zeta) \mathbf{n}) \cdot \vartheta_{0} \mathrm{~d} s=\int_{\partial \mathscr{B}_{\varepsilon}}(\sigma(\mathscr{H}) \mathbf{n}) \cdot \vartheta_{0} \mathrm{~d} s-\int_{\partial \mathscr{B}_{\varepsilon}}(\sigma(\zeta) \mathbf{n}) \cdot \vartheta_{0} \mathrm{~d} s .
$$

Firstly, we start by studying the integral $\int_{\partial \mathscr{B}_{\varepsilon}}(\sigma(\mathscr{H}) \mathbf{n}) \cdot \vartheta_{0} \mathrm{~d} s$. Expanding

$$
\vartheta_{0}(\varepsilon y)=\vartheta_{0}(0)+\varepsilon \nabla \vartheta_{0}\left(\tau_{y}\right) \quad \text { with } \tau_{y} \in \mathscr{B}_{\varepsilon},
$$

we obtain

$$
\int_{\partial \mathscr{B}_{\varepsilon}}(\sigma(\mathscr{H}) \mathbf{n}) \cdot \vartheta_{0} \mathrm{~d} s=\varepsilon \int_{\partial \mathscr{B}_{\varepsilon}}(\sigma(\mathscr{H}) \mathbf{n}) \cdot \nabla \vartheta_{0}\left(\tau_{y}\right) \mathrm{d} s+\int_{\partial \mathscr{B}_{\varepsilon}}(\sigma(\mathscr{H}) \mathbf{n}) \cdot \vartheta_{0}(0) \mathrm{d} s .
$$

Since $\nabla \vartheta_{0}$ is uniformly bounded in $\mathscr{B}_{\varepsilon}$, we see that

$$
\int_{\partial \mathscr{B}_{\varepsilon}}(\sigma(\mathscr{H}) \mathbf{n}) \cdot \vartheta_{0} \mathrm{~d} s=\left(\int_{\partial \mathscr{B}_{\varepsilon}}(\sigma(\mathscr{H}) \mathbf{n}) \mathrm{d} s\right) \cdot \vartheta_{0}(0)+O(\varepsilon) .
$$

On the other hand, we have

$$
\int_{\partial \mathscr{B}_{\varepsilon}}(\sigma(\mathscr{H}) \mathbf{n}) \mathrm{d} s=-\int_{\mathscr{B}_{\varepsilon}}-\operatorname{div} \sigma(\mathscr{H}) \mathrm{d} x=\frac{-1}{\beta} \psi_{0}(0) .
$$


Consequently,

$$
\int_{\partial \mathscr{B}_{\varepsilon}}(\sigma(\mathscr{H}) \mathbf{n}) \cdot \vartheta_{0} \mathrm{~d} s=\frac{-1}{\beta} \psi_{0}(0) \cdot \vartheta_{0}(0)+O(\varepsilon) .
$$

Similarly, we obtain

$$
\int_{\partial \mathscr{B}_{\varepsilon}}(\sigma(\zeta) \mathbf{n}) \cdot \vartheta_{0} \mathrm{~d} s=\left(\int_{\partial \mathscr{B}_{\varepsilon}}(\sigma(\zeta) \mathbf{n}) \mathrm{d} s\right) \cdot \vartheta_{0}(0)+O(\varepsilon) .
$$

From 4.8, we deduce that $-\operatorname{div} \sigma(\zeta)=0$ in $\mathscr{B}_{z, \varepsilon}$ and using the Green's formula, we obtain

$$
\int_{\partial \mathscr{B}_{\varepsilon}} \sigma(\zeta) \mathbf{n} \mathrm{d} s=\int_{\mathscr{B}_{\varepsilon}} \operatorname{div} \sigma(\zeta) \mathrm{d} x=0
$$

Therefore,

$$
\int_{\partial \mathscr{B}_{\varepsilon}}(\sigma(\zeta) \mathbf{n}) \cdot \vartheta_{0} \mathrm{~d} s=O(\varepsilon) .
$$

Inserting 4.20 and 4.21 into 4.19), we obtain

$$
\int_{\partial \mathscr{B}_{\varepsilon}}(\sigma(\mathscr{H}-\zeta) \mathbf{n}) \cdot \vartheta_{0} \mathrm{~d} s=\frac{-1}{\beta} \psi_{0}(0) \cdot \vartheta_{0}(0)+O(\varepsilon) .
$$

Gathering 4.17, 4.18) and 4.22 also from the definition of $\beta$ (see 4.7), we deduce the claimed expansion:

$$
\int_{\partial \mathscr{B}_{\varepsilon}} \sigma\left(\psi_{0}-\psi_{\varepsilon}\right) \mathbf{n} \cdot \vartheta_{0} \mathrm{~d} s=\frac{1}{-\log (\varepsilon)} \frac{4 \pi \mu(\mu+\eta)}{2 \mu+\eta} \psi_{0}(0) \cdot \vartheta_{0}(0)+o\left(\frac{1}{-\log (\varepsilon)}\right) .
$$

We present in the following lemma the asymptotic expansion of the last term on the right-hand side of 4.5 .

Lemma 4.9. The fourth term on the right-hand side of 4.5 admits the asymptotic expansion

$$
\int_{\Omega_{\varepsilon}} \sigma\left(\psi_{\varepsilon}-\psi_{0}\right): e\left(\psi_{\varepsilon}-\psi_{0}\right) \mathrm{d} x=\frac{1}{-\log (\varepsilon)} \frac{4 \pi \mu(\mu+\eta)}{2 \mu+\eta}\left|\psi_{0}(0)\right|^{2}+o\left(\frac{1}{-\log (\varepsilon)}\right)
$$

where the constant $\eta$ is defined in 4.16.

Proof. One can easily remark that $\psi_{\varepsilon}-\psi_{0}$ is the solution to the following boundary value problem:

$$
\begin{cases}-\operatorname{div} \sigma\left(\psi_{\varepsilon}-\psi_{0}\right)=0 & \text { in } \Omega_{\varepsilon}, \\ \psi_{\varepsilon}-\psi_{0}=0 & \text { on } \Gamma, \\ \sigma\left(\psi_{\varepsilon}-\psi_{0}\right) \mathbf{n}=0 & \text { on } \Sigma, \\ \psi_{\varepsilon}-\psi_{0}=-\psi_{0} & \text { on } \partial \mathscr{B}_{\varepsilon} .\end{cases}
$$


Using Green formula, we have

$$
\int_{\Omega_{\varepsilon}} \sigma\left(\psi_{\varepsilon}-\psi_{0}\right): e\left(\psi_{\varepsilon}-\psi_{0}\right) \mathrm{d} x=\int_{\partial \mathscr{B}_{\varepsilon}}\left(\sigma\left(\psi_{0}-\psi_{\varepsilon}\right) \mathbf{n}\right) \cdot \psi_{0} \mathrm{~d} x
$$

Applying the same technique developed in the proof of Lemma 4.8 , one can derive

$$
\int_{\partial \mathscr{B}_{\varepsilon}}\left(\sigma\left(\psi_{0}-\psi_{\varepsilon}\right) \mathbf{n}\right) \cdot \psi_{0} \mathrm{~d} x=\frac{1}{-\log (\varepsilon)} \frac{4 \pi \mu(\mu+\eta)}{2 \mu+\eta}\left|\psi_{0}(0)\right|^{2}+o\left(\frac{1}{-\log (\varepsilon)}\right) .
$$

Therefore,

$$
\int_{\Omega_{\varepsilon}} \sigma\left(\psi_{\varepsilon}-\psi_{0}\right): e\left(\psi_{\varepsilon}-\psi_{0}\right) \mathrm{d} x=\frac{1}{-\log (\varepsilon)} \frac{4 \pi \mu(\mu+\eta)}{2 \mu+\eta}\left|\psi_{0}(0)\right|^{2}+o\left(\frac{1}{-\log (\varepsilon)}\right) .
$$

\subsubsection{Asymptotic expansion}

We are now ready to present the main results of this section. Based on the estimate Lemmas 4.3, 4.4, 4.8 and 4.9, we deduce the following topological asymptotic expansion of the shape functional $\mathscr{J}$.

Proposition 4.10. We have

$$
\mathscr{J}\left(\Omega_{\varepsilon}\right)=\mathscr{J}(\Omega)+\frac{1}{-\log (\varepsilon)} \frac{4 \pi \mu(\mu+\eta)}{2 \mu+\eta}\left(\psi_{0}(0) \cdot \vartheta_{0}(0)+\left|\psi_{0}(0)\right|^{2}\right)+o\left(\frac{1}{-\log (\varepsilon)}\right) .
$$

\subsection{Sensitivity analysis of the Kohn-Vogelius functional}

In this section, we consider the Kohn-Vogelius functional $\mathscr{K}$. Recall that $\mathscr{K}$ measures the difference between the Dirichlet and Neumann solutions. In the presence of a small inclusion $\mathscr{B}_{z, \varepsilon}$ in $\Omega, \mathscr{K}$ is defined by

$$
\mathscr{K}\left(\Omega \backslash \overline{\mathscr{B}_{z, \varepsilon}}\right)=\int_{\Omega \backslash \overline{\mathscr{B}_{z, \varepsilon}}} \sigma\left(\psi_{\varepsilon}^{D}-\psi_{\varepsilon}^{N}\right): e\left(\psi_{\varepsilon}^{D}-\psi_{\varepsilon}^{N}\right) \mathrm{d} x,
$$

where $\psi_{\varepsilon}^{N} \in H^{1}(\Omega) / \mathscr{V}$ and $\psi_{\varepsilon}^{D} \in H^{1}(\Omega)$ solve respectively the problems $\mathscr{P}_{N}^{\varepsilon}$ and $\mathscr{P}_{D}^{\varepsilon}$.

The aim here is to derive an asymptotic expansion for $\mathscr{K}$ with respect to the presence of a small inclusion $\mathscr{B}_{z, \varepsilon}$ in $\Omega$. In order to derive a topological asymptotic expansion for the functional $\mathscr{K}$, we start our analysis by the following simplified variation of $\mathscr{K}$.

\subsubsection{Variation of the Kohn-Vogelius functional}

We present in this section the variation of the functional $\mathscr{K}$ with respect to the presence of a small inclusion $\mathscr{B}_{z, \varepsilon}$ inside the domain $\Omega$. We will derive a simplified expression of the variation $\mathscr{K}(\Omega \backslash \overline{\mathscr{B} z, \varepsilon})-\mathscr{K}(\Omega)$. The obtained result is presented in the following theorem. 
Theorem 4.11. We have

$$
\mathscr{K}\left(\Omega \backslash \overline{\mathscr{B}_{z, \varepsilon}}\right)-\mathscr{K}(\Omega)=\mathrm{A}_{N}(\varepsilon)+\mathrm{A}_{D}(\varepsilon),
$$

where

$$
\mathrm{A}_{N}(\varepsilon):=\int_{\partial \mathscr{B}_{z, \varepsilon}}\left(\sigma\left(\psi_{\varepsilon}^{N}-\psi_{0}^{N}\right) \mathbf{n}\right) \cdot \psi_{0}^{N} \mathrm{~d} s-\int_{\mathscr{B}_{z, \varepsilon}} \sigma\left(\psi_{0}^{N}\right): e\left(\psi_{0}^{N}\right) \mathrm{d} x
$$

and

$$
\mathrm{A}_{D}(\varepsilon):=-3 \int_{\mathscr{B}_{z, \varepsilon}} \sigma\left(\psi_{0}^{D}\right): e\left(\psi_{0}^{D}\right) \mathrm{d} x-\int_{\partial \mathscr{B}_{z, \varepsilon}}\left(\sigma\left(\psi_{\varepsilon}^{D}-\psi_{0}^{D}\right) \mathbf{n}\right) \cdot \psi_{0}^{D} \mathrm{~d} s .
$$

Proof. The Kohn-Vogelius functional $\mathscr{K}$ can be splitted into three terms

$$
\mathscr{K}\left(\Omega \backslash \overline{\mathscr{B}_{z, \varepsilon}}\right)=\mathscr{K}_{D D}\left(\Omega \backslash \overline{\mathscr{B}_{z, \varepsilon}}\right)-2 \mathscr{K}_{N D}\left(\Omega \backslash \overline{\mathscr{B}_{z, \varepsilon}}\right)+\mathscr{K}_{N N}\left(\Omega \backslash \overline{\mathscr{B}_{z, \varepsilon}}\right)
$$

where

$$
\begin{aligned}
& \mathscr{K}_{D D}\left(\Omega \backslash \overline{\mathscr{B}_{z, \varepsilon}}\right)=\int_{\Omega \backslash \overline{\mathscr{B}_{z, \varepsilon}}} \sigma\left(\psi_{\varepsilon}^{D}\right): e\left(\psi_{\varepsilon}^{D}\right) \mathrm{d} x, \\
& \mathscr{K}_{N D}\left(\Omega \backslash \overline{\mathscr{B}_{z, \varepsilon}}\right)=\int_{\Omega \backslash \overline{\mathscr{B}_{z, \varepsilon}}} \sigma\left(\psi_{\varepsilon}^{N}\right): e\left(\psi_{\varepsilon}^{D}\right) \mathrm{d} x, \\
& \mathscr{K}_{N N}\left(\Omega \backslash \overline{\mathscr{B}_{z, \varepsilon}}\right)=\int_{\Omega \backslash \overline{\mathscr{B}_{z, \varepsilon}}} \sigma\left(\psi_{\varepsilon}^{N}\right): e\left(\psi_{\varepsilon}^{N}\right) \mathrm{d} x .
\end{aligned}
$$

- Variation of $\mathscr{K}_{D D}$. We have

$$
\begin{aligned}
& \mathscr{K}_{D D}\left(\Omega \backslash \overline{\mathscr{B}_{z, \varepsilon}}\right)-\mathscr{K}_{D D}(\Omega) \\
= & \int_{\Omega \backslash \mathscr{\mathscr { B }}_{z, \varepsilon}} \sigma\left(\psi_{\varepsilon}^{D}\right): e\left(\psi_{\varepsilon}^{D}\right) \mathrm{d} x-\int_{\Omega} \sigma\left(\psi_{0}^{D}\right): e\left(\psi_{0}^{D}\right) \mathrm{d} x \\
= & \int_{\Omega \backslash \overline{\mathscr{B}_{z, \varepsilon}}} \sigma\left(\psi_{\varepsilon}^{D}-\psi_{0}^{D}\right): e\left(\psi_{\varepsilon}^{D}-\psi_{0}^{D}\right) \mathrm{d} x+2 \int_{\Omega \backslash \overline{\mathscr{B}_{z, \varepsilon}}} \sigma\left(\psi_{\varepsilon}^{D}-\psi_{0}^{D}\right): e\left(\psi_{0}^{D}\right) \mathrm{d} x \\
& -\int_{\mathscr{B}_{z, \varepsilon}} \sigma\left(\psi_{0}^{D}\right): e\left(\psi_{0}^{D}\right) \mathrm{d} x .
\end{aligned}
$$

Using Green's formula in the two first integrals, we obtain

$$
\begin{aligned}
\int_{\Omega \backslash \mathscr{B}_{z, \varepsilon}} \sigma\left(\psi_{\varepsilon}^{D}-\psi_{0}^{D}\right): e\left(\psi_{\varepsilon}^{D}-\psi_{0}^{D}\right) \mathrm{d} x & =-\int_{\partial \mathscr{B}_{z, \varepsilon}}\left(\sigma\left(\psi_{\varepsilon}^{D}-\psi_{0}^{D}\right) \mathbf{n}\right) \cdot \psi_{0}^{D} \mathrm{~d} s \\
\int_{\Omega \backslash \mathscr{B}_{z, \varepsilon}} \sigma\left(\psi_{\varepsilon}^{D}-\psi_{0}^{D}\right): e\left(\psi_{0}^{D}\right) \mathrm{d} x & =\int_{\partial \mathscr{B}_{z, \varepsilon}}\left(\sigma\left(\psi_{0}^{D}\right) \mathbf{n}\right) \cdot\left(\psi_{\varepsilon}^{D}-\psi_{0}^{D}\right) \mathrm{d} s \\
& =-\int_{\partial \mathscr{B}_{z, \varepsilon}}\left(\sigma\left(\psi_{0}^{D}\right) \mathbf{n}\right) \cdot \psi_{0}^{D} \mathrm{~d} s \\
& =-\int_{\mathscr{B}_{z, \varepsilon}} \sigma\left(\psi_{0}^{D}\right): e\left(\psi_{0}^{D}\right) \mathrm{d} x
\end{aligned}
$$


Then, it follows that $\mathscr{K}_{D D}\left(\Omega \backslash \overline{\mathscr{B}_{z, \varepsilon}}\right)-\mathscr{K}_{D D}(\Omega)=-\int_{\partial \mathscr{B}_{z, \varepsilon}}\left(\sigma\left(\psi_{\varepsilon}^{D}-\psi_{0}^{D}\right) \mathbf{n}\right) \cdot \psi_{0}^{D} \mathrm{~d} s-3 \int_{\mathscr{B}_{z, \varepsilon}} \sigma\left(\psi_{0}^{D}\right): e\left(\psi_{0}^{D}\right) \mathrm{d} x$.

- Variation of $\mathscr{K}_{N D}$. Again using Green formula on $\left(\sqrt{\mathscr{P}_{N}^{\varepsilon}}\right)$ and $\left(\sqrt{\mathscr{P}_{N}^{0}}\right)$, we get

$$
\begin{aligned}
\mathscr{K}_{N D}\left(\Omega \backslash \overline{\mathscr{B}_{z, \varepsilon}}\right)-\mathscr{K}_{N D}(\Omega) & =\int_{\Omega \backslash \overline{\mathscr{B}_{z, \varepsilon}}} \sigma\left(\psi_{\varepsilon}^{N}\right): e\left(\psi_{\varepsilon}^{D}\right) \mathrm{d} x-\int_{\Omega} \sigma\left(\psi_{0}^{N}\right): e\left(\psi_{0}^{D}\right) \mathrm{d} x \\
& =\int_{\partial \Omega}\left(\sigma\left(\psi_{\varepsilon}^{N}\right) \mathbf{n}\right) \cdot \psi_{\varepsilon}^{D} \mathrm{~d} s-\int_{\partial \Omega}\left(\sigma\left(\psi_{0}^{N}\right) \mathbf{n}\right) \cdot \psi_{0}^{D} \mathrm{~d} s .
\end{aligned}
$$

- Variation of $\mathscr{K}_{N N}$. We have

$$
\begin{aligned}
& \mathscr{K}_{N N}\left(\Omega \backslash \overline{\mathscr{B}_{z, \varepsilon}}\right)-\mathscr{K}_{N N}(\Omega) \\
= & \int_{\Omega \backslash \mathscr{B}_{z, \varepsilon}} \sigma\left(\psi_{\varepsilon}^{N}\right): e\left(\psi_{\varepsilon}^{N}\right) \mathrm{d} x-\int_{\Omega} \sigma\left(\psi_{0}^{N}\right): e\left(\psi_{0}^{N}\right) \mathrm{d} x \\
= & \int_{\Omega \backslash \overline{\mathscr{B}_{z, \varepsilon}}} \sigma\left(\psi_{\varepsilon}^{N}-\psi_{0}^{N}\right): e\left(\psi_{\varepsilon}^{N}\right) \mathrm{d} x+\int_{\Omega_{z, \varepsilon}} \sigma\left(\psi_{\varepsilon}^{N}-\psi_{0}^{N}\right): e\left(\psi_{\varepsilon}^{N}\right) \mathrm{d} x \\
& -\int_{\mathscr{B}_{z, \varepsilon}} \sigma\left(\psi_{0}^{N}\right): e\left(\psi_{0}^{N}\right) \mathrm{d} x .
\end{aligned}
$$

Moreover, using Green formula, we have

$$
\begin{aligned}
\mathscr{K}_{N N}\left(\Omega \backslash \overline{\mathscr{B}_{z, \varepsilon}}\right)-\mathscr{K}_{N N}(\Omega)= & \int_{\partial \Omega}\left(\sigma\left(\psi_{\varepsilon}^{N}-\psi_{0}^{N}\right) \mathbf{n}\right) \cdot \psi_{\varepsilon}^{N} \mathrm{~d} s+\int_{\partial \Omega}\left(\sigma\left(\psi_{\varepsilon}^{N}-\psi_{0}^{N}\right) \mathbf{n}\right) \cdot \psi_{0}^{N} \mathrm{~d} s \\
& +\int_{\partial \mathscr{B}_{z, \varepsilon}}\left(\sigma\left(\psi_{\varepsilon}^{N}-\psi_{0}^{N}\right) \mathbf{n}\right) \cdot \psi_{0}^{N} \mathrm{~d} s-\int_{\mathscr{B}_{z, \varepsilon}} \sigma\left(\psi_{0}^{N}\right): e\left(\psi_{0}^{N}\right) \mathrm{d} x .
\end{aligned}
$$

Combining the variations of $\mathscr{K}_{D D}, \mathscr{K}_{N D}$, and $\mathscr{K}_{N N}$ the Kohn-Vogelius functional $\mathscr{K}$ has the following variation

$$
\begin{aligned}
& \mathscr{K}\left(\Omega \backslash \overline{\mathscr{B}_{z, \varepsilon}}\right)-\mathscr{K}(\Omega) \\
= & \int_{\partial \mathscr{B}_{z, \varepsilon}}\left(\sigma\left(\psi_{\varepsilon}^{N}-\psi_{0}^{N}\right) \mathbf{n}\right) \cdot \psi_{0}^{N} \mathrm{~d} s-\int_{\partial \mathscr{B}_{z, \varepsilon}}\left(\sigma\left(\psi_{\varepsilon}^{D}-\psi_{0}^{D}\right) \mathbf{n}\right) \cdot \psi_{0}^{D} \mathrm{~d} s \\
& -3 \int_{\mathscr{B}_{z, \varepsilon}} \sigma\left(\psi_{0}^{D}\right): e\left(\psi_{0}^{D}\right) \mathrm{d} x-\int_{\mathscr{B}_{z, \varepsilon}} \sigma\left(\psi_{0}^{N}\right): e\left(\psi_{0}^{N}\right) \mathrm{d} x \\
& -2 \int_{\partial \Omega}\left(\sigma\left(\psi_{\varepsilon}^{N}\right) \mathbf{n}\right) \cdot \psi_{\varepsilon}^{D} \mathrm{~d} s+2 \int_{\partial \Omega}\left(\sigma\left(\psi_{0}^{N}\right) \mathbf{n}\right) \cdot \psi_{0}^{D} \mathrm{~d} s \\
& +\int_{\partial \Omega}\left(\sigma\left(\psi_{\varepsilon}^{N}-\psi_{0}^{N}\right) \mathbf{n}\right) \cdot \psi_{\varepsilon}^{N} \mathrm{~d} s+\int_{\partial \Omega}\left(\sigma\left(\psi_{\varepsilon}^{N}-\psi_{0}^{N}\right) \mathbf{n}\right) \cdot \psi_{0}^{N} \mathrm{~d} s .
\end{aligned}
$$

Since $\psi_{\varepsilon}^{D}=\psi_{0}^{D}=\mathscr{U}_{d}$ on $\partial \Omega$ and $\sigma\left(\psi_{\varepsilon}^{N}\right) \mathbf{n}=\sigma\left(\psi_{0}^{N}\right) \mathbf{n}=\varphi$ on $\partial \Omega$, we prove that

$$
\int_{\partial \Omega}\left(\sigma\left(\psi_{\varepsilon}^{N}\right) \mathbf{n}\right) \cdot \psi_{\varepsilon}^{D} \mathrm{~d} s-\int_{\partial \Omega}\left(\sigma\left(\psi_{0}^{N}\right) \mathbf{n}\right) \cdot \psi_{0}^{D} \mathrm{~d} s=\int_{\partial \Omega} \varphi \cdot \mathscr{U}_{d} \mathrm{~d} s-\int_{\partial \Omega} \varphi \cdot \mathscr{U}_{d} \mathrm{~d} s=0
$$


and

$$
\int_{\partial \Omega}\left(\sigma\left(\psi_{\varepsilon}^{N}-\psi_{0}^{N}\right) \mathbf{n}\right) \cdot \psi_{\varepsilon}^{N} \mathrm{~d} s=0 \quad \text { and } \quad \int_{\partial \Omega}\left(\sigma\left(\psi_{\varepsilon}^{N}-\psi_{0}^{N}\right) \mathbf{n}\right) \cdot \psi_{0}^{N} \mathrm{~d} s=0 .
$$

Then by combining the above equalities we obtain the desired result.

Remark 4.12. The variation of the terms $\mathscr{K}_{D D}, \mathscr{K}_{N D}$ and $\mathscr{K}_{N N}$ can be calculated using the established results in Section 4.1. But in this case the topological gradient expression will be dependent on the solution of the adjoint state.

\subsubsection{Asymptotic expansion of the Kohn-Vogelius functional}

We are now in position to state the main result of this section. Based on the previous variation of $\mathscr{K}$, we derive a topological asymptotic expansion which is summarized in the following theorem.

Theorem 4.13. For $z \in \Omega$, the Kohn-Vogelius functional $\mathscr{K}$ admits the following asymptotic expansion:

$$
\mathscr{K}\left(\Omega \backslash \overline{\mathscr{B}_{z, \varepsilon}}\right)=\mathscr{K}(\Omega)+\frac{1}{-\log (\varepsilon)} \delta \mathscr{K}(z)+o\left(\frac{1}{-\log (\varepsilon)}\right)
$$

with $\delta \mathscr{K}$ being the topological gradient given by

$$
\delta \mathscr{K}(x)=\frac{4 \pi \mu(\mu+\eta)}{2 \mu+\eta}\left(\left|\psi_{0}^{D}(x)\right|^{2}-\left|\psi_{0}^{N}(x)\right|^{2}\right), \quad \forall x \in \Omega,
$$

where $\psi_{0}^{N}$ and $\psi_{0}^{D}$ solve the problems $\left(\mathscr{P}_{N}^{0}\right.$ and $\left[\mathscr{P}_{D}^{0}\right.$ respectively and the constant $\eta$ is defined in (4.16).

Proof. We recall that we will detail the proof only for the case of an origin-centered inclusion (see Remark 3.3).

From Theorem 4.11, we have

$$
\mathscr{K}\left(\Omega \backslash \overline{\mathscr{B}_{\varepsilon}}\right)-\mathscr{K}(\Omega)=\mathrm{A}_{N}(\varepsilon)+\mathrm{A}_{D}(\varepsilon)
$$

Next, we will derive an asymptotic expansion of $\mathrm{A}_{N}(\varepsilon)$ and $\mathrm{A}_{D}(\varepsilon)$.

Asymptotic expansion of $\mathrm{A}_{N}(\varepsilon)$ : We have

$$
\mathrm{A}_{N}(\varepsilon)=\int_{\partial \mathscr{B}_{\varepsilon}}\left(\sigma\left(\psi_{\varepsilon}^{N}-\psi_{0}^{N}\right) \mathbf{n}\right) \cdot \psi_{0}^{N} \mathrm{~d} s-\int_{\mathscr{B}_{\varepsilon}} \sigma\left(\psi_{0}^{N}\right): e\left(\psi_{0}^{N}\right) \mathrm{d} x .
$$

Now, we study each term of $\mathrm{A}_{N}(\varepsilon)$ separately. Let us first focus on the first term of $\mathrm{A}_{N}(\varepsilon)$. We have

$$
\int_{\partial \mathscr{B}_{\varepsilon}}\left(\sigma\left(\psi_{0}^{N}-\psi_{\varepsilon}^{N}\right) \mathbf{n}\right) \cdot \psi_{0}^{N} \mathrm{~d} s=\int_{\partial \mathscr{B}_{\varepsilon}}\left(\sigma\left(r_{\varepsilon}^{N}\right) \mathbf{n}\right) \cdot \psi_{0}^{N} \mathrm{~d} s-\mathrm{T}_{\varepsilon} \int_{\partial \mathscr{B}_{\varepsilon}}\left(\sigma\left(\mathscr{H}^{N}-\zeta^{N}\right) \mathbf{n}\right) \cdot \psi_{0}^{N} \mathrm{~d} s
$$


with $r_{\varepsilon}^{N}=\psi_{0}^{N}-\psi_{\varepsilon}^{N}+\mathrm{T}_{\varepsilon}\left(\mathscr{H}^{N}-\zeta^{N}\right)$ where $\mathscr{H}^{N}(x)=\frac{-1}{\beta} G(x) \psi_{0}^{N}(0)$ and $\zeta^{N}$ solve the following value problem

$$
\begin{cases}-\operatorname{div} \sigma\left(\zeta^{N}\right)=0 & \text { in } \Omega \\ \zeta^{N}=\mathscr{H}^{N} & \text { on } \partial \Omega .\end{cases}
$$

Using the same technique described in the proof of Lemma 4.8, one can prove that

$$
\int_{\partial \mathscr{B}_{\varepsilon}}\left(\sigma\left(\psi_{0}^{N}-\psi_{\varepsilon}^{N}\right) \mathbf{n}\right) \cdot \psi_{0}^{N} \mathrm{~d} s=\frac{1}{-\log (\varepsilon)} \frac{4 \pi \mu(\mu+\eta)}{2 \mu+\eta}\left|\psi_{0}^{N}(0)\right|^{2}+o\left(\frac{1}{-\log (\varepsilon)}\right) .
$$

For the other term, we know using elliptic regularity that $\nabla \psi_{0}^{N}$ is uniformly bounded in $\mathscr{B}_{\varepsilon}$. Thus,

$$
\int_{\mathscr{B}_{\varepsilon}} \sigma\left(\psi_{0}^{N}\right): e\left(\psi_{0}^{N}\right) \mathrm{d} x \leq c \int_{\mathscr{C}} \varepsilon^{2}=O\left(\varepsilon^{2}\right)
$$

Therefore,

$$
\mathrm{A}_{N}(\varepsilon)=\frac{1}{-\log (\varepsilon)} \frac{4 \pi \mu(\mu+\eta)}{2 \mu+\eta}\left|\psi_{0}^{N}(0)\right|^{2}+o\left(\frac{1}{-\log (\varepsilon)}\right) .
$$

In a similar manner, we prove the following asymptotic expansion:

$$
\mathrm{A}_{D}(\varepsilon)=\frac{1}{-\log (\varepsilon)} \frac{4 \pi \mu(\mu+\eta)}{2 \mu+\eta}\left|\psi_{0}^{D}(0)\right|^{2}+o\left(\frac{1}{-\log (\varepsilon)}\right) .
$$

By combining the above equalities, we have

$$
\mathscr{K}\left(\Omega_{\varepsilon}\right)-\mathscr{K}(\Omega)=\frac{1}{-\log (\varepsilon)} \frac{4 \pi \mu(\mu+\eta)}{2 \mu+\eta}\left(\left|\psi_{0}^{D}(0)\right|^{2}-\left|\psi_{0}^{N}(0)\right|^{2}\right)+o\left(\frac{1}{-\log (\varepsilon)}\right) .
$$

\section{Numerical implementation}

This section is concerned with some numerical investigations. We start this section by a numerical validation of the topological asymptotic expansion established in Theorem 4.13 . After that, we give some reconstruction results obtained by a one-iteration algorithm showing the efficiency and accuracy of our approach: only one iteration is needed to reconstruct an unknown rigid inclusion immersed in an elastic material from over-determined boundary measurements.

In our numerical examples, we use the following setting and parameters:

- The computational domain $\Omega$ is defined by the square $\Omega=(0,1) \times(0,1)$.

- The measurement $\mathscr{U}_{d}$ is reconstructed using a synthetic data: we fix a shape $\mathscr{B}^{*}$, solve the linear elasticity problem 2.1 in $\Omega \backslash \overline{\mathscr{B}^{*}}$ and extract the measurement $\mathscr{U}_{d}$ by computing $\psi$ on $\partial \Omega$.

- Concerning the mesh, we impose a fixed number of discretization points for the boundary $\partial \Omega$, that is 100 mesh points in each direction (i.e., $h=1 / 100$ ).

The numerical simulations is implemented using the free software FreeFem ++ . 


\subsection{Numerical validation}

It is proved in Section 5.1 that the variation of the Kohn-Vogelius function $\mathscr{K}$, with respect to the presence of a small arbitrary shape of a rigid inclusion, admits the following asymptotic expansion:

$$
\mathscr{K}\left(\Omega_{z, \varepsilon}\right)-\mathscr{K}(\Omega)=\frac{1}{-\log (\varepsilon)} \delta \mathscr{K}(z)+o\left(\frac{1}{-\log (\varepsilon)}\right),
$$

where $\delta \mathscr{K}$ is the topological gradient defined by

$$
\delta \mathscr{K}(z)=\frac{4 \pi \mu(2 \mu+\lambda)}{3 \mu+\lambda}\left(\left|\psi_{0}^{D}(z)\right|^{2}-\left|\psi_{0}^{N}(z)\right|^{2}\right), \quad \forall z \in \Omega .
$$

Here, we give a numerical validation of this asymptotic behavior. To this end, we simulate the variation of the following function:

$$
\Delta_{z}(\varepsilon)=\mathscr{K}\left(\Omega_{z, \varepsilon}\right)-\mathscr{K}(\Omega)+\frac{1}{\log (\varepsilon)} \delta \mathscr{K}(z), \quad \forall z \in \Omega
$$

with respect to $-1 / \log (\varepsilon)$ for some spherical inclusions $\mathscr{B}_{z_{i}, \varepsilon}=z_{i}+\varepsilon \mathrm{B}(0,1), i=1, \ldots, 4$, created in the unit square $\Omega$. The locations $z_{i}$ of the considered rigid inclusions $\mathscr{B}_{z_{i}, \varepsilon}$ are described in Table 5.1. We expect to prove numerically that $\Delta_{z_{i}}(\varepsilon)$ satisfies the obtained theoretical estimate

$$
\Delta_{z_{i}}(\varepsilon)=o\left(\frac{1}{-\log (\varepsilon)}\right) \text {. }
$$

\begin{tabular}{|c|c|c|c|c|}
\hline Inclusion $\mathscr{B}_{z_{i}, \varepsilon}$ & $\mathscr{B}_{z_{1}, \varepsilon}$ & $\mathscr{B}_{z_{2}, \varepsilon}$ & $\mathscr{B}_{z_{3}, \varepsilon}$ & $\mathscr{B}_{z_{4}, \varepsilon}$ \\
\hline Location $z_{i}$ & $(0.4,0.4)$ & $(0.5,0.1)$ & $(0.7,0.3)$ & $(0.9,0.2)$ \\
\hline
\end{tabular}

Table 5.1: Coordinates the inclusions centers $z_{i}, i=1, \ldots, 4$.

From the expression of $\Delta_{z}(\varepsilon)$, we adopt the following numerical procedure.

\section{Numerical validation algorithm:}

- Step 1:

- Solve the problems $\left(\mathscr{P}_{D}^{0}\right)$ and $\left(\mathscr{P}_{N}^{0}\right)$ in $\Omega$.

- Compute $\mathscr{K}(\Omega)$ (see $(3.1))$.

- Step 2: For each inclusion $\mathscr{B}_{z_{i}, \varepsilon}=z_{i}+\varepsilon B(0,1), i=1, \ldots, 4$ :

- Determine the variation $\delta \mathscr{K}\left(z_{i}\right)$ defined in 5.2$)$.

- Choose $\varepsilon_{0}^{i}=\max \left\{\varepsilon>0\right.$, such that $\left.z_{i}+\varepsilon_{0}^{i} B(0,1) \subset \Omega\right\}$.

- Compute an approximation of the function $\left.\left.\varepsilon \mapsto \mathscr{K}\left(\Omega \backslash \overline{\mathscr{B}_{z_{i}, \varepsilon}}\right), \varepsilon \in\right] 0, \varepsilon_{0}^{i}\right]$. 
- Step 3: Deduce a numerical approximation of the function $\varepsilon \mapsto \log \left(\left|\Delta_{z_{i}}(\varepsilon)\right|\right)$ with respect to $\log (-\log (\varepsilon))$.

The obtained results are illustrated in Figure 5.1. For each inclusions $\mathscr{B}_{z_{i}, \varepsilon}, i=1, \ldots, 4$, we numerically compute the variation of the function $\varepsilon \mapsto \log \left(\left|\Delta_{z_{i}}(\varepsilon)\right|\right)$ with respect to $\log (-\log (\varepsilon))$.
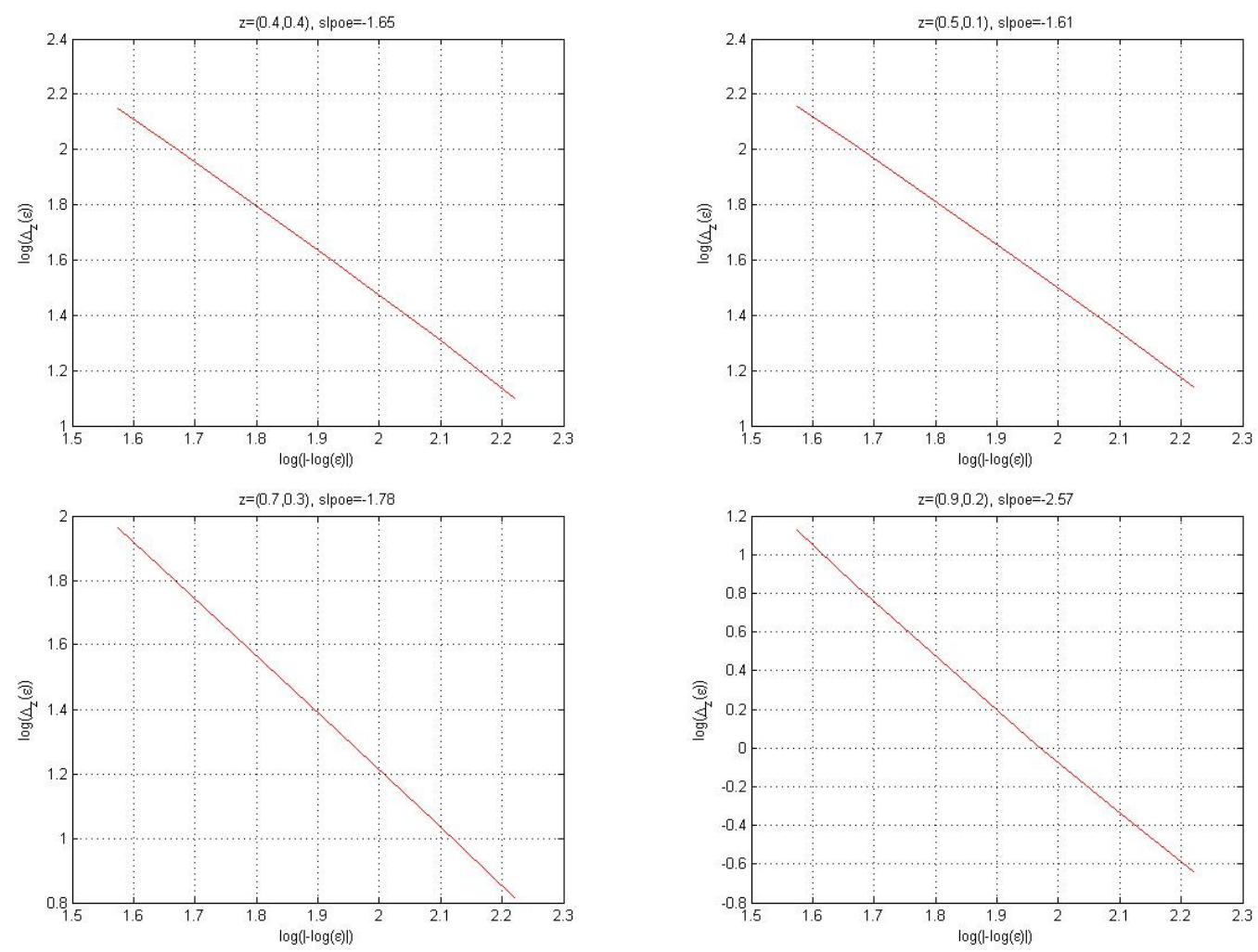

Figure 5.1: Variation of $\log \left(\left|\Delta_{z_{i}}(\varepsilon)\right|\right)$ with respect to $\log (-\log (\varepsilon)), i=1, \ldots, 4$.

Denoting by $\alpha_{i}$ the unknown parameter describing the behavior of the function $\varepsilon \mapsto$ $\Delta_{z_{i}}(\varepsilon)$ with respect to $-\log (\varepsilon)$, i.e.,

$$
\left|\Delta_{z_{i}}(\varepsilon)\right|=O\left((-\log (\varepsilon))^{\alpha_{i}}\right)
$$

Then, one can observe that $\alpha_{i}$ can be characterized as the slope of the line approximating the variation $\varepsilon \mapsto \log \left(\left|\Delta_{z_{i}}(\varepsilon)\right|\right)$ with respect to $\log (-\log (\varepsilon))$.

From the presented curves in Figure 5.1, we deduce the slopes $\alpha_{i}, i=1, \ldots, 4$ (see Table 5.2 describing the behavior of the function $\varepsilon \mapsto \Delta_{z}(\varepsilon)$ with respect to $-\log (\varepsilon)$. 


\begin{tabular}{|c|c|c|c|c|}
\hline Inclusion $\mathscr{B}_{z_{i}, \varepsilon}$ & $\mathscr{B}_{z_{1}, \varepsilon}$ & $\mathscr{B}_{z_{2}, \varepsilon}$ & $\mathscr{B}_{z_{3}, \varepsilon}$ & $\mathscr{B}_{z_{4}, \varepsilon}$ \\
\hline The slope $\alpha_{i}$ & $\alpha_{1}=-1.65$ & $\alpha_{2}=-1.61$ & $\alpha_{3}=-1.78$ & $\alpha_{4}=-2.57$ \\
\hline
\end{tabular}

Table 5.2: The slopes $\alpha_{i}, i=1, \ldots, 4$ of the line approximating the variation of the function $\log \left(\left|\Delta_{z_{i}}(\varepsilon)\right|\right)$ with respect to $\log (-\log (\varepsilon))$.

From Table 5.2, for each considered inclusion $\mathscr{B}_{z_{i}, \varepsilon}$, one can observe that the obtained slope $\alpha_{i}$ satisfies the inequality: $\alpha_{i}<-1, i=1, \ldots, 4$, which confirm the behavior predicted by the theoretical result

$$
\Delta_{z_{i}}(\varepsilon)=o\left(\frac{1}{-\log (\varepsilon)}\right), \quad i=1, \ldots, 4 .
$$

\subsection{Reconstruction results}

In this section, we propose an accurate and efficient non-iterative algorithm for reconstructing an unknown rigid inclusion. The proposed procedure based on the asymptotic expansion 4.23). The rigid inclusion $\mathscr{B}^{*}$ is located and reconstructed using a level-set curve of the topological gradient $\delta \mathscr{K}$. The main steps of the proposed procedure are described in the following algorithm.

\section{The one-shot Algorithm.}

1. Solve the unperturbed problems $\left(\mathscr{P}_{N}^{0}\right)$ and $\left(\mathscr{P}_{D}^{0}\right)$ in $\Omega$.

2. Compute the topological gradient $\delta \mathscr{K}$ defined in (5.1).

3. Determine the rigid inclusion $\mathscr{B}^{*}$.

The location of the rigid inclusion $\mathscr{B}^{*}$ is given by the point $z^{*} \in \Omega$ where the topological gradient $\delta \mathscr{K}$ is most negative. The size of $\mathscr{B}^{*}$ is approximated using numerical simulation. Let $\delta_{\text {min }}=\delta \mathscr{K}\left(z^{*}\right) \leq \delta \mathscr{K}(x), \forall x \in \Omega$, the rigid inclusion $\mathscr{B}^{*}$ is approximated as follows

$$
\mathscr{B}^{*}=\left\{x \in \Omega, \delta \mathscr{K}(x) \leq c^{*} \delta_{\min }\right\},
$$

where $c^{*} \in(0,1)$ such that

$$
\mathscr{K}\left(\Omega \backslash \overline{\mathscr{B}_{c^{*}}}\right) \leq \mathscr{K}\left(\Omega \backslash \overline{\mathscr{B}_{c}}\right), \quad \forall c \in(0,1)
$$

with $\mathscr{B}_{c}=\left\{x \in \Omega, \delta \mathscr{K}(x) \leq c \delta_{\min }\right\}$.

Remark 5.1. The idea to determine the constant $c$ is to calculate the minimum of the Hausdorff distance $d_{c}$ between $\mathscr{B}^{*}$ and $\mathscr{B}_{c}$

$$
d_{c}\left(\mathscr{B}^{*}, \mathscr{B}_{c}\right)=\frac{\operatorname{meas}\left(\mathscr{B}^{*} \cup \mathscr{B}_{c}\right)-\operatorname{meas}\left(\mathscr{B}^{*} \cap \mathscr{B}_{c}\right)}{\operatorname{meas}\left(\mathscr{B}^{*}\right)},
$$

where meas $(E)$ denotes the Lebesgue measure of the set $E \subset \Omega$. 
Next, we apply the proposed algorithm for solving some examples to demonstrate the efficiency of the proposed algorithm. Examples 5.2 5.5 are free of noise, while in Example 5.6 the measured data $\mathscr{U}_{d}$ is corrupted with noise.

Example 5.2 (Reconstruction of circular-shaped inclusion). In this example, we test our reconstruction algorithm to reconstruct an inclusion having a circular shape centered at $(0.5,0.5)$ with radius $r^{*}=0.1$. The obtained reconstruction results are presented in Figure 5.2 .

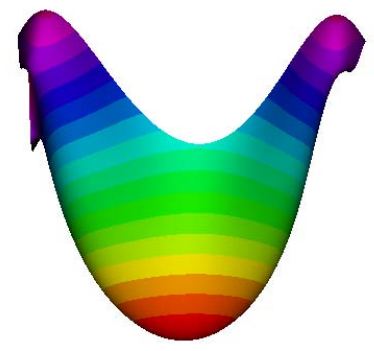

(a) Negative zone (zed zone) of $\delta \mathscr{K}$

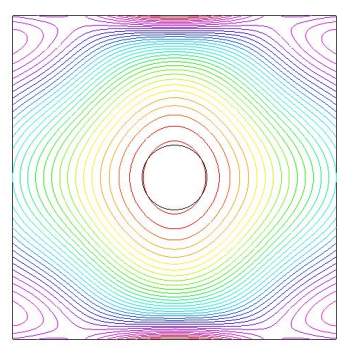

(b) Iso-values of $\delta \mathscr{K}$

Figure 5.2: Topological gradient $\delta \mathscr{K}$ in the presence of a circle shape.

In this first simulation, we show:

- The negative zone (red zone) described by the function $x \mapsto(x, \delta \mathscr{K}(x)), \forall x \in \Omega$, see Figure 5.2(a).

- The iso-values of the topological gradient function $\delta \mathscr{K}$ in the presence of the unknown boundary $\partial \mathscr{B}^{*}$ (circle black line), see Figure $5.2(\mathrm{~b})$.

As one can observe in Figure 5.2 , the unknown boundary $\partial \mathscr{B}^{*}$ (circle centered at $(0.5,0.5)$ with radius $\left.r^{*}=0.1\right)$ is located in the region where the topological gradient is the most negative and it is approximated by a level set curve of the topological gradient. The result is efficient and the reconstruction of circular shape is very close to the actual rigid inclusion.

To reconstruct the exact geometry of the unknown inclusion in Figure 5.2(b) (disc centered at $(0.5,0.5)$ with shared radius $\left.r^{*}=0.1\right)$, we minimize the error function of the Hausdorff distance $d_{c}$. In order to compute numerically an approximation of the minimum of the error function $d_{c}$, we divide the interval $[0,1]$ into $\mathrm{M}$ equal subintervals (i.e., of size $1 / \mathrm{M})$. We denote by $c_{i}=i / \mathrm{M}, 1 \leq i \leq \mathrm{M}$ the $(\mathrm{M}+1)$ endpoints of these intervals and we take $c^{*}=\arg \min _{c \in\left\{c_{1}, \ldots, c_{M}\right\}} d_{c}$. The reconstruction results are illustrated in Figures 5.3 and 5.4 . 


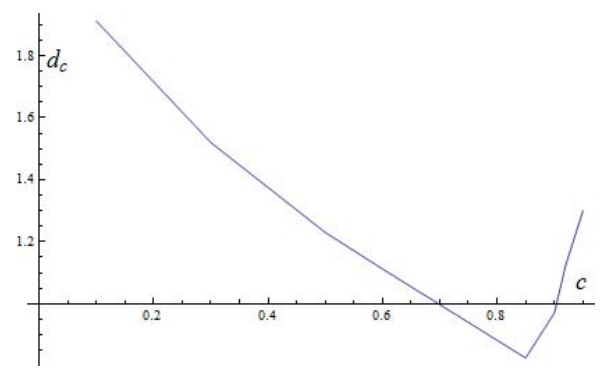

Figure 5.3: Variation of the error function $d_{c}$ with respect to $c$.

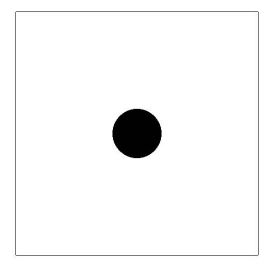

(a) Exact shape

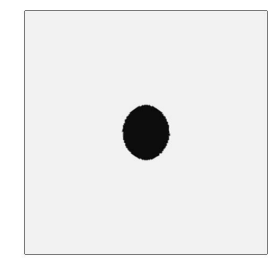

(b) Reconstruction with $c^{*}=0.85$

Figure 5.4: Reconstruction of circular shaped inclusion.

Example 5.3 (Reconstruction of ellipse-shaped inclusion). In this example, we examine the numerical reconstruction of unknown inclusion described by an ellipse centered at $(0.5,0.5)$. We represent the reconstruction result in Figure 5.5 where we see that the reconstruction is again efficient.

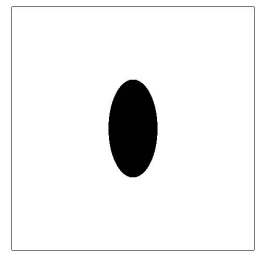

(a) Exact shape

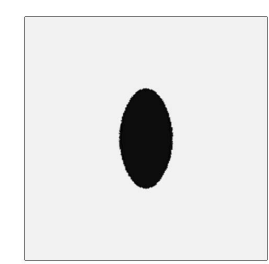

(b) Reconstruction with $c^{*}=0.065$

Figure 5.5: Reconstruction of an elliptical shaped.

In the next example, we prove that the computation of the topological gradient does not depend on the number of inclusions inside the domain $\Omega$.

Example 5.4 (Reconstruction of multiple inclusions). In this example, we want to reconstruct three discs $\mathscr{B}_{1}^{*}, \mathscr{B}_{2}^{*}$ and $\mathscr{B}_{3}^{*}$ centered respectively at $(0.3,0.7),(0.2,0.2)$ and $(0.8,0.8)$ with shared radius $r^{*}=0.02$. The obtained results are illustrated in Figure 5.6 . 
Here again, as one can see in Figure 5.6, the one-shot algorithm gives quite efficient reconstruction result for location ,size and number of rigid inclusions.

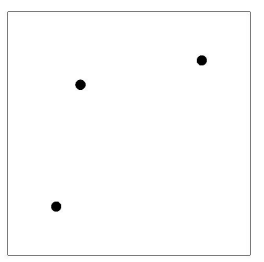

(a) Exact shape

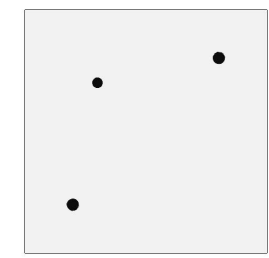

(b) Reconstruction with $c^{*}=0.92$

Figure 5.6: Reconstruction of multi-inclusions.

In conclusion of these first simulations, this approach permits to give us acceptable knowledge of the number, location and shape of inclusions. Moreover, it is efficient to detect different types of shapes such as circle and ellipse.

In the previous examples, we reconstructed an inclusion with a regular shape (circle and ellipse). Next we test our algorithm to reconstruct more complicated geometry.

Example 5.5 (Shape with corners). In the previous example, the reconstruction seems to be efficient to reconstruct simple rigid inclusions. We apply, now, the proposed algorithm to detect more complicated geometry. Our aim is to reconstruct geometry containing straight lines and corners from over-determined boundary data. More precisely, we want to detect a small square $\mathscr{B}^{*}=(0.4,0.6) \times(0.4,0.6)$. We present the reconstruction results in Figure 5.7. One can remark here, that the unknown rigid inclusion (square) is located but the boundary $\partial \mathscr{B}^{*}$ cannot be well approximated by any iso-value curve. Therefore, the obtained result can serve as a good initial guess in level-sets-based methods, for instance.

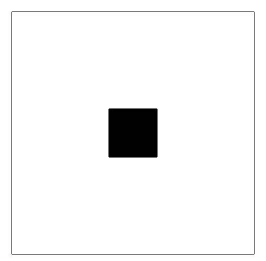

(a) Exact shape

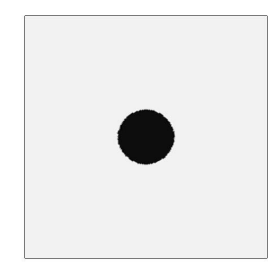

(b) Reconstruction with $c^{*}=0.73$

Figure 5.7: Reconstruction of an inclusion with corner.

Example 5.6 (Reconstruction with noisy data). Now the synthetic boundary measure- 
ment $\mathscr{U}_{d}$ is corrupted with noise. The noisy measurement is generated by

$$
\mathscr{U}_{d}^{\rho}(x)=(1+\rho \times \text { rand }) \times \mathscr{U}_{d}(x), \quad x \in \partial \Omega,
$$

where $\rho$ indicates the noise level of the measurement and the function rand generates a random number with the uniform distribution over $(-1,1)$. The idea is to test the stability of the one-shot algorithm with respect to noisy data.

For this test, we reconstruct two ellipses $\mathscr{B}_{1}^{*}$ and $\mathscr{B}_{2}^{*}$ centered respectively at $(0.15,0.5)$ and $(0.85,0.5)$. The obtained results are illustrated in Figure 5.8. From the reconstruction results in Figure 5.8, we can notice that if the noise level $\rho$ is no more than $10 \%$, that our algorithm is able to detect, with precision, the location and the shape of rigid inclusion, whereas for a noise level larger than 15\%, fictitious inclusions show up.

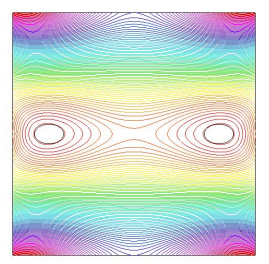

(a) Iso-values of $\delta \mathscr{K}$ with $\rho=0 \%$

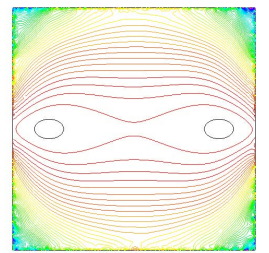

(e) Iso-values of $\delta \mathscr{K}$ with $\rho=10 \%$

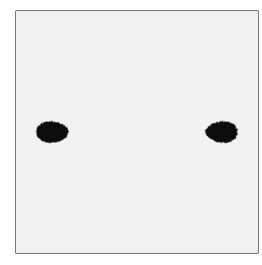

(b) $c^{*}=0.00082$ and $\rho=0 \%$

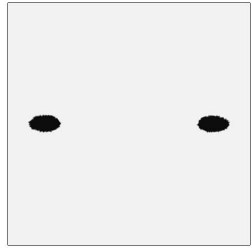

(f) $c^{*}=0.00082$ and $\rho=10 \%$

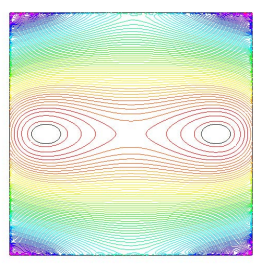

(c) Iso-values of $\delta \mathscr{K}$ with $\rho=5 \%$

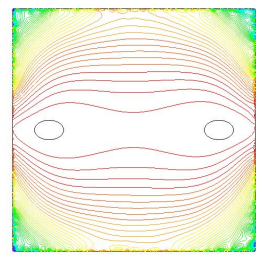

(g) Iso-values of $\delta \mathscr{K}$ with $\rho=15 \%$

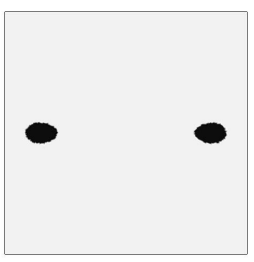

(d) $c^{*}=0.00082$ and $\rho=5 \%$

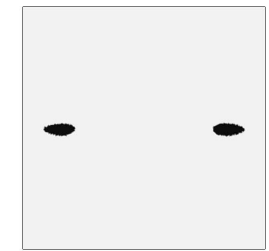

(h) $c^{*}=0.00082$ and $\rho=15 \%$

Figure 5.8: Reconstruction with noise data.

\section{Conclusion}

The presented paper concerns the detection of location and shape of a rigid inclusions inside an elastic body from a single pair Cauchy data in two dimensional case. We have proposed a non-iterative reconstruction algorithm based on the topological sensitivity analysis method combined with the so-called Kohn-Vogelius type functional. The main idea consists in reformulate the inverse problem as an optimization problem, where a Kohn-Vogelius functional is minimized in the class of admissible geometries inclusions. A topological sensitivity analysis is derived for the Kohn-Vogelius functional. The obtained 
an asymptotic formula valid for any arbitrary geometric perturbations. From these results, a new non-iterative reconstruction algorithm has been devised. Particularly, we test the efficiency and accuracy of the proposed procedure by some parameters such as the number, the shape, the location, and the size of the inclusions.

\section{Proofs}

In this section, we prove Lemma 4.7. We start by presenting some definitions and preliminary lemmas used to prove this lemma.

\subsection{Definitions and notations}

Definition 7.1. Let $\mathscr{O}$ be a bounded, connected, and open subset in $\mathbb{R}^{d}(d \in\{2,3\})$ with a Lipschitz continuous boundary $\partial \mathscr{O}$.

- The Sobolev space $H^{1}(\mathscr{O})$ is equipped with the norm

$$
\|\psi\|_{1, \mathscr{O}}^{2}=\int_{\mathscr{O}} \sigma(\psi): e(\psi)+\psi \cdot \psi \mathrm{d} x
$$

which, due to the Korn's inequality [36, Lemma 5.4.4], is equivalent to the usual norm. We will also need the semi-norm defined by

$$
|\psi|_{1, \mathscr{O}}^{2}=\int_{\mathscr{O}} \sigma(\psi): e(\psi) \mathrm{d} x .
$$

Moreover, for a given function $\psi \in H^{1}(\mathscr{O})$, we define the function $\widetilde{\psi}$ on $\widetilde{\mathscr{O}}:=\mathscr{O} / \varepsilon$ by

$$
\widetilde{\psi}(y)=\psi(x), \quad y=x / \varepsilon .
$$

Using that $e_{i j}(\psi)=\frac{1}{2}\left(\frac{\partial \psi_{i}}{\partial x_{j}}+\frac{\partial \psi_{j}}{\partial x_{i}}\right)$ and $\nabla_{x} \psi(x)=\left(\nabla_{y} \psi(y)\right) / \varepsilon$, we obtain

$$
|\psi|_{1, \mathscr{O}}^{2}=\int_{\mathscr{O}} \sigma(\psi): e(\psi) \mathrm{d} x=\frac{1}{\varepsilon^{2}} \int_{\widetilde{\mathscr{O}}} \sigma(\widetilde{\psi}): e(\widetilde{\psi}) \varepsilon^{2} \mathrm{~d} y .
$$

Hence,

$$
|\psi|_{1, \mathscr{O}}=|\widetilde{\psi}|_{1, \widetilde{\mathscr{O}}}
$$

Similarly, we have

$$
\|\psi\|_{0, \mathscr{O}}=\varepsilon\|\widetilde{\psi}\|_{0, \widetilde{\mathscr{O}}}
$$

- The trace norm $\|\cdot\|_{1 / 2, \partial \mathscr{B}_{z, \varepsilon}}$ is defined by

$$
\|g\|_{1 / 2, \partial \mathscr{B}_{z, \varepsilon}}=\inf \left\{\|\psi\|_{H^{1}\left(\Omega \backslash \overline{\mathscr{B}_{z, \varepsilon}}\right)} ; \psi \in H^{1}\left(\mathscr{O} \backslash \overline{\mathscr{B}_{z, \varepsilon}}\right), \psi=g \text { on } \partial \mathscr{B}_{z, \varepsilon}\right\}
$$


In the following definition, we recall the weighted Sobolev spaces and we introduce the weight function $\kappa(x):=\left(2+|x|^{2}\right)^{1 / 2}$.

Definition 7.2. Let $1<p<\infty$. For each real number $\rho$ and each open set $\mathscr{O} \subset \mathbb{R}^{d}$, we set

$$
\begin{aligned}
L_{\rho}^{p}(\mathscr{O}) & :=\left\{\psi \in \mathscr{D}^{\prime}(\mathscr{O}), \kappa^{\rho} \psi \in L^{p}(\mathscr{O})\right\}, \\
\mathrm{W}_{\rho}^{1, p}(\mathscr{O}) & := \begin{cases}\left\{\psi \in \mathscr{D}^{\prime}(\mathscr{O}), \psi \in L_{\rho-1}^{p}(\mathscr{O}), \nabla \psi \in L_{\rho}^{p}(\mathscr{O})\right\} & \text { if } \frac{d}{p}+\rho \neq 1, \\
\left\{\psi \in \mathscr{D}^{\prime}(\mathscr{O}), \frac{\psi}{\log (\varepsilon)} \in L_{\rho-1}^{p}(\mathscr{O}), \nabla \psi \in L_{\rho}^{p}(\mathscr{O})\right\} & \text { if } \frac{d}{p}+\rho=1 .\end{cases}
\end{aligned}
$$

We define now the space $\mathrm{W}_{\rho}^{1, p}(\mathscr{O})=\left\{\psi \in \mathrm{W}_{\rho}^{1, p}(\mathscr{O}), \psi=0\right.$ on $\left.\partial \mathscr{O}\right\}$. The dual space of $\mathrm{W}_{\rho}^{1, p}(\mathscr{O})$ is denoted by $\mathrm{W}_{-\rho}^{-1, p^{\prime}}(\mathscr{O})$, where $p^{\prime}$ is such that $1 / p+1 / p^{\prime}=1$ (it is a subspace of $\left.\mathscr{D}^{\prime}(\mathscr{O})\right)$. Notice that $L_{\rho}^{p}(\mathscr{O})$ and $\mathrm{W}_{\rho}^{1, p}(\mathscr{O})$ are reflexive Banach spaces with respect to the norms:

$$
\begin{aligned}
\|\psi\|_{L_{\rho}^{p}(\mathscr{O})} & :=\left\|\kappa^{\rho} \psi\right\|_{L^{p}(\mathscr{O})}, \\
\|\psi\|_{\mathrm{W}_{\rho}^{1, p}(\mathscr{O})} & := \begin{cases}\left(\|\psi\|_{L_{\rho-1}^{p}(\mathscr{O})}^{p}+\|\nabla \psi\|_{L_{\rho}^{p}(\mathscr{O})}^{p}\right)^{1 / p} & \text { if } \frac{d}{p}+\rho \neq 1 \\
\left(\left\|\frac{\psi}{\log (\kappa)}\right\|_{L_{\rho-1}^{p}(\mathscr{O})}^{p}+\|\nabla \psi\|_{L_{\rho}^{p}(\mathscr{O})}^{p}\right)^{1 / p} & \text { if } \frac{d}{p}+\rho=1 .\end{cases}
\end{aligned}
$$

We refer to [7] for more details about the previous Sobolev spaces.

Next, we recall a result concerning domains depending on a parameter.

\subsection{Preliminary lemmas}

Theorem 7.3. [47, Section 4.1.3, p. 214] Let $\Omega$ and $\mathscr{C}$ be two bounded simply connected domains of $\mathbb{R}^{d}(d \in\{2,3\})$ of class $C^{0,1}$. Let $p \in(1,+\infty), \varepsilon \in(0,1 / 2)$ and $\mathscr{B}_{z, \varepsilon}=z+\varepsilon \mathscr{C}$. Let us assume that $\overline{\mathscr{B}_{z, \varepsilon}} \subset \Omega$ and that there exists a constant $c>0$ depending only of $d$, $p, \mathscr{C}$ and $\Omega$ such that $\operatorname{dist}\left(\mathscr{B}_{z, \varepsilon}, \partial \Omega\right)>c \varepsilon$. Then

$$
\langle\cdot\rangle_{p, \partial \mathscr{B}_{z, \varepsilon}} \sim b(\varepsilon)\|\cdot\|_{\mathrm{L}^{p}\left(\partial \mathscr{B}_{z, \varepsilon}\right)}+[\cdot]_{p, \partial \mathscr{B}_{z, \varepsilon}}
$$

where

$$
\begin{gathered}
\langle g\rangle_{p, \partial \mathscr{B}_{z, \varepsilon}}=\inf \left\{\|\psi\|_{\mathrm{W}^{1, p}\left(\Omega \backslash \overline{\mathscr{B}_{z, \varepsilon}}\right.} ; \psi \in \mathrm{W}^{1, p}\left(\Omega \backslash \overline{\mathscr{B}_{z, \varepsilon}}\right), \psi=g \text { on } \partial \mathscr{B}_{z, \varepsilon}\right\}, \\
b(\varepsilon)= \begin{cases}\varepsilon^{(1-d) / p} \min \left(1, \varepsilon^{d / p-1}\right) & \text { if } p<d, \\
\varepsilon^{(1-d) / p} \min \left(1,|\log (\varepsilon)|^{(1-p) / p}\right) & \text { if } p=d, \\
\varepsilon^{(1-d) / p} & \text { if } p>d,\end{cases}
\end{gathered}
$$


and

$$
\begin{aligned}
{[g]_{1, \partial \mathscr{B}_{z, \varepsilon}} } & =\frac{1}{\left|\partial \mathscr{B}_{z, \varepsilon}\right|} \iint_{\partial \mathscr{B}_{z, \varepsilon} \times \partial \mathscr{B}_{z, \varepsilon}}|g(x)-g(y)| \mathrm{d} s(x) \mathrm{d} s(y), \\
{[g]_{p, \partial \mathscr{B}_{z, \varepsilon}} } & =\left(\iint_{\partial \mathscr{B}_{z, \varepsilon} \times \partial \mathscr{B}_{z, \varepsilon}} \frac{|g(x)-g(y)|^{p}}{|x-y|^{d+p-2}} \mathrm{~d} s(x) \mathrm{d} s(y)\right)^{1 / p} \quad \text { for } p \in(1,+\infty) .
\end{aligned}
$$

The next lemma concerns the linear elasticity problem in the whole space $\mathbb{R}^{2}$.

Lemma 7.4. Let $\psi$ be a solution of

$$
-\operatorname{div} \sigma(\psi)=0 \quad \text { in } \mathbb{R}^{2}
$$

Then every solution which is a tempered distribution should be a polynomial.

Proof. Applying Fourier transform to 7.4 we immediately notice that the support of $\widehat{\psi}$ is contained in $\{0\}$. Therefore, those distributions should be a finite sum of Dirac deltas. By the inverse Fourier transform we deduce that $\psi$ is polynomial.

The next lemma gives a decomposition of the exterior linear elasticity problem.

Lemma 7.5. Let $\mathscr{C}$ be a Lipschitz open set of $\mathbb{R}^{2}$ and let $\gamma \in H^{1 / 2}(\partial \mathscr{C})$. We consider $\psi \in \mathrm{W}_{0}^{1,2}\left(\mathbb{R}^{2} \backslash \overline{\mathscr{C}}\right)$ the solution of the exterior elasticity problem

$$
\begin{cases}-\operatorname{div} \sigma(\psi)=0 & \text { in } \mathbb{R}^{2} \backslash \overline{\mathscr{C}} \\ \psi=\gamma & \text { on } \partial \mathscr{C} .\end{cases}
$$

Then there exists a constant $\varrho \in \mathbb{R}^{2}$ such that $\psi=\varrho+W_{\mathscr{C}}$ with $W_{\mathscr{C}}(y)=O(1 / r)$ and

$$
|\varrho| \leq c\|\gamma\|_{1 / 2, \partial \mathscr{C}}
$$

with $c>0$ is a constant.

Proof. Let $\phi \in \mathscr{D}\left(\mathbb{R}^{2}\right)$, we have

$$
\langle-\operatorname{div} \sigma(\psi), \phi\rangle_{\mathscr{D}, \mathscr{D}^{\prime}, \mathbb{R}^{2}}=\int_{\partial \mathscr{C}}[\sigma(\psi) \mathbf{n}] \cdot \phi \mathrm{d} s .
$$

Then,

$$
-\operatorname{div} \sigma(\psi)=[\sigma(\psi) \mathbf{n}] \delta_{\partial \mathscr{C}}:=T \quad \text { in } \mathscr{D}^{\prime}\left(\mathbb{R}^{2}\right)
$$

Now, let us define

$$
u=G * T,
$$

where $*$ denotes the convolution product and $G$ is the fundamental solution of the elasticity problem given by 4.6 . Therefore,

$$
-\operatorname{div} \sigma(u)=T \quad \text { in } \mathscr{D}^{\prime}\left(\mathbb{R}^{2}\right)
$$


Now notice that the variable $w=\psi-u$ solve the problem $(7.5)$, then by Lemma 7.4 the solution $w$ of 7.5 should be a polynomial. Therefore,

$$
\psi=G * T+\varrho=\int_{\partial \mathscr{C}} l(x) G(y-x) \mathrm{d} s(x)+P,
$$

where $P$ is polynomial and $l(x)=\sigma(v) \mathbf{n}(x)$.

Using a Taylor development of $G$, we obtain

$$
G(y-x)=G(y)-\nabla G(\xi(y, x)) x,
$$

where $\xi(y, x)=y-v x$ with $v \in(0,1)$, then

$$
\psi=G(y) \int_{\partial \mathscr{C}} l(x) \mathrm{d} s(x)-\int_{\partial \mathscr{C}} l(x) \nabla G(\xi(y, x)) \mathrm{d} s(x)+P .
$$

On the other hand, we know that $\log \notin \mathrm{W}_{0}^{1,2}\left(\mathbb{R}^{2} \backslash \overline{\mathscr{C}}\right)$, it follows that

$$
\int_{\partial \mathscr{C}} l(x) \mathrm{d} s(x)=0 .
$$

Also, due to $P \in \mathrm{W}_{0}^{1,2}\left(\mathbb{R}^{2} \backslash \overline{\mathscr{C}}\right)$, we must have

$$
P=\varrho \text { with } \varrho \text { is a constant in } \mathbb{R}^{2} .
$$

Therefore,

$$
\psi(y)=\varrho-\int_{\partial \mathscr{C}} l(x) \nabla G(\xi(y, x)) \mathrm{d} s(x)=\varrho+W_{\mathscr{C}}(y)
$$

and $\psi$ is bounded at infinity. Moreover, we have $W_{\mathscr{C}}(y)=O(1 / r)$ (see [26]) and there exist $c>0$ such that

$$
|\varrho| \leq c\|\gamma\|_{1 / 2, \partial \mathscr{C}}
$$

Lemma 7.6. Let $\theta \in H^{1 / 2}(\partial \mathscr{C})$ such that $\int_{\partial \mathscr{C}} \theta \mathrm{d} s=0$, let $\psi \in \mathrm{W}_{0}^{1,2}\left(\mathbb{R}^{2} \backslash \overline{\mathscr{C}}\right)$ be the solution to the problem

$$
\begin{cases}-\operatorname{div} \sigma(\psi)=0 & \text { in } \mathbb{R}^{2} \backslash \overline{\mathscr{C}} \\ \psi=\theta & \text { on } \partial \mathscr{C} .\end{cases}
$$

From the previous lemma $\psi$ decomposed as follow $\psi=\rho+W_{\mathscr{C}}$. Then there exist a constant $c>0$ and $\varepsilon_{1}>0$ such that for all $0<\varepsilon<\varepsilon_{1}$ :

$$
\left\|W_{\mathscr{C}}\right\|_{0, \Omega_{R} / \varepsilon} \leq c\|\theta\|_{1 / 2, \partial \mathscr{C}} \quad \text { and } \quad\left|W_{\mathscr{C}}\right|_{1, \Omega_{R} / \varepsilon} \leq c \varepsilon^{2}\|\theta\|_{1 / 2, \partial \mathscr{C}}
$$

Proof. Following the same lines as the proof in [29, Lemma 4.1] we can prove 7.6 .

The next lemma is a particular case of Lemma 4.7 . 
Lemma 7.7. Let $\varepsilon>0$. For $\theta_{N} \in H^{-1 / 2}(\Sigma), \theta_{D} \in H^{1 / 2}(\Gamma)$ and $\varrho \in \mathbb{R}^{2}$, let $v_{\varepsilon} \in$ $H^{1}\left(\Omega \backslash \overline{\mathscr{B}_{z, \varepsilon}}\right)$ be the solution of the linear elasticity problem

$$
\begin{cases}-\operatorname{div} \sigma\left(v_{\varepsilon}\right)=0 & \text { in } \Omega \backslash \overline{\mathscr{B}_{z, \varepsilon}}, \\ \sigma\left(v_{\varepsilon}\right) \mathbf{n}=\theta_{N} & \text { on } \Sigma, \\ v_{\varepsilon}=\theta_{D} & \text { on } \Gamma, \\ v_{\varepsilon}=\varrho & \text { on } \partial \mathscr{B}_{z, \varepsilon} .\end{cases}
$$

There exists a constant $c>0$ (independent of $\varepsilon$ ) such that

$$
\left\|v_{\varepsilon}\right\|_{1, \Omega \backslash \overline{\mathscr{B}_{z, \varepsilon}}} \leq c\left(\left\|\theta_{N}\right\|_{-1 / 2, \Sigma}+\left\|\theta_{D}\right\|_{1 / 2, \Gamma}+|\varrho|\right) .
$$

Proof. For simplicity, we denote by $\Omega_{z, \varepsilon}=\Omega \backslash \overline{\mathscr{B}_{z, \varepsilon}}$. Let $\varepsilon>0$ and $v_{\varepsilon} \in H^{1}\left(\Omega_{z, \varepsilon}\right)$ be the solution of the value problem (7.7). Let $Z_{\varepsilon} \in H^{1}\left(\Omega_{z, \varepsilon}\right)$ be the solution of

$$
\begin{cases}-\operatorname{div} \sigma\left(Z_{\varepsilon}\right)=0 & \text { in } \Omega_{z, \varepsilon}, \\ \sigma\left(Z_{\varepsilon}\right) \mathbf{n}=0 & \text { on } \Sigma, \\ Z_{\varepsilon}=\theta_{D} & \text { on } \Gamma, \\ Z_{\varepsilon}=\varrho & \text { on } \partial \mathscr{B}_{z, \varepsilon} .\end{cases}
$$

Let $\widetilde{v}_{\varepsilon}$ and $\widetilde{Z}_{\varepsilon}$ be the respective extensions of $v_{\varepsilon}$ and $Z_{\varepsilon}$ to $\Omega$ by $\varrho$. From $(7.7)$ and 7.8 , we have

$$
\int_{\Omega_{z, \varepsilon}} \sigma\left(v_{\varepsilon}-Z_{\varepsilon}\right): e(\Phi) \mathrm{d} x=\left\langle\theta_{N}, \Phi\right\rangle_{-1 / 2,1 / 2, \Sigma} \quad \text { for all } \Phi \in H_{\Gamma}^{1}\left(\Omega_{z, \varepsilon}\right),
$$

where

$$
H_{\Gamma}^{1}\left(\Omega_{z, \varepsilon}\right)=\left\{w \in H^{1}\left(\Omega_{z, \varepsilon}\right), w=0 \text { on } \Gamma \text { and } w=0 \text { on } \partial \mathscr{B}_{z, \varepsilon}\right\} .
$$

By taking $\Phi=v_{\varepsilon}-Z_{\varepsilon}$ as test function, we obtain

$$
\left|\widetilde{v}_{\varepsilon}-\widetilde{Z}_{\varepsilon}\right|_{1, \Omega}^{2}=\left\langle\theta_{N}, v_{\varepsilon}-Z_{\varepsilon}\right\rangle_{-1 / 2,1 / 2, \Sigma}
$$

Then, from the trace theorem there exists a constant $c>0$ (independent of $\varepsilon$ ) such that

$$
\left|\widetilde{v}_{\varepsilon}-\widetilde{Z}_{\varepsilon}\right|_{1, \Omega}^{2} \leq c\left\|\theta_{N}\right\|_{-1 / 2, \Sigma}\left\|v_{\varepsilon}-Z_{\varepsilon}\right\|_{1 / 2, \Sigma} \leq c\left\|\theta_{N}\right\|_{-1 / 2, \Sigma}\left\|\widetilde{v}_{\varepsilon}-\widetilde{Z}_{\varepsilon}\right\|_{1, \Omega} .
$$

Moreover, using that $v_{\varepsilon}-Z_{\varepsilon}=0$ on $\Gamma$ and Korn's inequality we get

$$
\left\|\widetilde{v}_{\varepsilon}-\widetilde{Z}_{\varepsilon}\right\|_{1, \Omega} \leq c\left|\widetilde{v}_{\varepsilon}-\widetilde{Z}_{\varepsilon}\right|_{1, \Omega} .
$$

Hence,

$$
\begin{aligned}
\left\|v_{\varepsilon}-Z_{\varepsilon}\right\|_{1, \Omega_{z, \varepsilon}}^{2}=\left\|\widetilde{v}_{\varepsilon}-\widetilde{Z}_{\varepsilon}\right\|_{1, \Omega}^{2} & \leq c\left\|\theta_{N}\right\|_{-1 / 2, \Sigma}\left\|\widetilde{v}_{\varepsilon}-\widetilde{Z}_{\varepsilon}\right\|_{1, \Omega} \\
& \leq c\left\|\theta_{N}\right\|_{-1 / 2, \Sigma}\left\|v_{\varepsilon}-Z_{\varepsilon}\right\|_{1, \Omega_{z, \varepsilon}} .
\end{aligned}
$$


Therefore, we have

$$
\left\|v_{\varepsilon}-Z_{\varepsilon}\right\|_{1, \Omega_{z, \varepsilon}} \leq c\left\|\theta_{N}\right\|_{-1 / 2, \Sigma} .
$$

Now, we prove $\left\|Z_{\varepsilon}\right\|_{1, \Omega_{z, \varepsilon}} \leq c\left(\left\|\theta_{D}\right\|_{1 / 2, \Gamma}+|\varrho|\right)$. For a fixed $\varepsilon_{0}>0$, the problem (7.7) is well-posed and admits a unique solution $Z_{\varepsilon} \in H^{1}\left(\Omega_{z, \varepsilon_{0}}\right)$ and there exists a constant $c>0$ such that

$$
\left\|Z_{\varepsilon}\right\|_{1, \Omega_{z, \varepsilon_{0}}} \leq c\left(\left\|\theta_{D}\right\|_{1 / 2, \Gamma}+\|\varrho\|_{1 / 2, \partial \mathscr{B}_{z, \varepsilon_{0}}}\right) .
$$

Using Theorem 7.3 , we get that

$$
\|\varrho\|_{1 / 2, \partial \mathscr{B}_{z, \varepsilon_{0}}} \sim \frac{1}{\left(\varepsilon_{0}\left(-\log \left(\varepsilon_{0}\right)\right)\right)^{1 / 2}}\|\varrho\|_{\mathrm{L}^{2}\left(\partial \mathscr{B}_{z, \varepsilon_{0}}\right)}+[\varrho]_{1 / 2, \partial \mathscr{B}_{z, \varepsilon_{0}}} .
$$

Using that $\varrho$ is constant and by a change of variables, we have

$$
\|\varrho\|_{1 / 2, \partial \mathscr{B}_{z, \varepsilon_{0}}} \sim \frac{1}{\left(\varepsilon_{0}\left(-\log \left(\varepsilon_{0}\right)\right)\right)^{1 / 2}}\|\varrho\|_{L^{2}\left(\partial \mathscr{B}_{z, \varepsilon_{0}}\right)}=\frac{1}{\left(-\log \left(\varepsilon_{0}\right)\right)^{1 / 2}}\|\varrho\|_{L^{2}(\partial \mathscr{C})}=c|\varrho|
$$

with $c$ being a constant depend of $\varepsilon_{0}$ and $\partial \mathscr{C}$.

Then, one can deduce

$$
\left\|Z_{\varepsilon}\right\|_{1, \Omega_{z, \varepsilon_{0}}} \leq c\left(\left\|\theta_{D}\right\|_{1 / 2, \Gamma}+|\varrho|\right)
$$

Let $0<\varepsilon_{1}<\varepsilon_{0}$ such that $\Omega_{z, \varepsilon_{0}} \subset \Omega_{z, \varepsilon}$ for all $0<\varepsilon<\varepsilon_{1}$. We denote by $\widetilde{Z}_{\varepsilon_{0}}$ the extension of $Z_{\varepsilon_{0}}$ to $\Omega$ by $\varrho$. Notice that the solution $Z_{\varepsilon}$ of the problem $(7.7)$ can be considered as the solution of the following minimization problem:

$$
\min _{Z \in \Theta}\left\{|Z|_{1, \Omega_{z, \varepsilon}}\right\}
$$

where the functional space $\Theta$ is defined as

$$
\Theta=\left\{Z \in H^{1}\left(\Omega_{z, \varepsilon}\right), Z=\varrho \text { on } \partial \mathscr{B}_{z, \varepsilon}, Z=\theta_{D} \text { on } \Gamma\right\}
$$

Therefore, for all $0<\varepsilon<\varepsilon_{1}$, we have

$$
\left|Z_{\varepsilon}\right|_{1, \Omega_{z, \varepsilon}} \leq c\left|\widetilde{Z}_{\varepsilon_{0}}\right|_{1, \Omega_{z, \varepsilon}}=c\left|Z_{\varepsilon_{0}}\right|_{1, \Omega_{z, \varepsilon_{0}}} \leq c\left\|Z_{\varepsilon_{0}}\right\|_{1, \Omega_{z, \varepsilon_{0}}} .
$$

Inserting 7.9 into 7.10 , we deduce

$$
\left|Z_{\varepsilon}\right|_{1, \Omega_{z, \varepsilon}} \leq c\left(\left\|\theta_{D}\right\|_{1 / 2, \Gamma}+|\varrho|\right) .
$$

Using the Korn's inequality, it follows that

$$
\begin{aligned}
\left\|Z_{\varepsilon}\right\|_{0, \Omega_{z, \varepsilon}} & =\left\|\widetilde{Z}_{\varepsilon}\right\|_{0, \Omega} \leq\left\|\widetilde{Z}_{\varepsilon}-Z_{0}\right\|_{0, \Omega}+\left\|Z_{0}\right\|_{0, \Omega} \leq c\left|\widetilde{Z}_{\varepsilon}-Z_{0}\right|_{1, \Omega}+\left\|Z_{0}\right\|_{0, \Omega} \\
& \leq c\left|\widetilde{Z}_{\varepsilon}\right|_{1, \Omega}+c\left\|Z_{0}\right\|_{1, \Omega} \leq c\left(\left|Z_{\varepsilon}\right|_{1, \Omega_{z, \varepsilon}}+\left\|Z_{0}\right\|_{1, \Omega}\right)
\end{aligned}
$$


Notice that $Z_{0}$ satisfies

$$
\begin{cases}-\operatorname{div} \sigma\left(Z_{0}\right)=0 & \text { in } \Omega, \\ \sigma\left(Z_{0}\right) \mathbf{n}=0 & \text { on } \Sigma, \\ Z_{0}=\theta_{D} & \text { on } \Gamma\end{cases}
$$

From the weak formulation of the value problem $(7.13)$ and we take $Z_{0}$ as test function, we deduce

$$
\left\|Z_{0}\right\|_{1, \Omega} \leq c\left\|\theta_{D}\right\|_{1 / 2, \Gamma}
$$

Inserting (7.11) and 7.14) into 7.12 , we obtain

$$
\left\|Z_{\varepsilon}\right\|_{0, \Omega_{z, \varepsilon}} \leq c\left(\left\|\theta_{D}\right\|_{1 / 2, \Gamma}+|\varrho|\right) .
$$

Then, by combining the equalities 7.11 and 7.15 , we get

$$
\left\|Z_{\varepsilon}\right\|_{1, \Omega_{z, \varepsilon}} \leq c\left(\left\|\theta_{D}\right\|_{1 / 2, \Gamma}+|\varrho|\right) .
$$

Therefore, we finally get

$$
\left\|v_{\varepsilon}\right\|_{1, \Omega_{z, \varepsilon}} \leq\left\|v_{\varepsilon}-Z_{\varepsilon}\right\|_{1, \Omega_{z, \varepsilon}}+\left\|Z_{\varepsilon}\right\|_{1, \Omega_{z, \varepsilon}} \leq c\left(\left\|\theta_{N}\right\|_{-1 / 2, \Sigma}+\left\|\theta_{D}\right\|_{1 / 2, \Gamma}+|\varrho|\right) .
$$

Now, we are ready to prove Lemma 4.7 .

\subsection{Proof of Lemma 4.7}

The proof of Lemma 4.7 is decomposed in the following two cases.

First case if $\theta$ is constant on $\partial \mathscr{B}_{z, \varepsilon}$, the previous lemma gives the desired result.

Second case if $\theta$ is not constant. Let $Z$ the solution of

$$
\begin{cases}-\operatorname{div} \sigma(Z)=0 & \text { in } \mathbb{R}^{2} \backslash \overline{\mathscr{B}_{z, \varepsilon}} \\ Z=\theta & \text { on } \partial \mathscr{B}_{z, \varepsilon} .\end{cases}
$$

From Lemma 7.5 , we have $Z=\varrho+W_{\mathscr{C}}$ with $\varrho \in \mathbb{R}^{2}$ and $W_{\mathscr{C}}(y)=O(1 / r)$. We define $w_{\varepsilon}=v_{\varepsilon}-W_{\mathscr{C}}\left(\frac{x-z}{\varepsilon}\right)$ with $y=(x-z) / \varepsilon$. Notice that $w_{\varepsilon}$ satisfies

$$
\begin{cases}-\operatorname{div} \sigma\left(w_{\varepsilon}\right)=0 & \text { in } \Omega_{z, \varepsilon}, \\ \sigma\left(w_{\varepsilon}\right) \mathbf{n}=\theta_{N}-\sigma\left(W_{\mathscr{C}}\left(\frac{x-z}{\varepsilon}\right)\right) \mathbf{n} & \text { on } \Sigma, \\ w_{\varepsilon}=\theta_{D}-W_{\mathscr{C}}\left(\frac{x-z}{\varepsilon}\right) & \text { on } \Gamma, \\ w_{\varepsilon}=\varrho & \text { on } \partial \mathscr{B}_{z, \varepsilon},\end{cases}
$$


where $\Omega_{z, \varepsilon}=\Omega \backslash \overline{\mathscr{B}} z, \varepsilon$. Using the previous lemma there exists a constant $c>0$ independent of $\varepsilon$ such that

$$
\begin{aligned}
& \left\|w_{\varepsilon}\right\|_{1, \Omega_{z, \varepsilon}} \\
\leq & c\left(\left\|\theta_{N}-\sigma\left(W_{\mathscr{C}}\left(\frac{x-z}{\varepsilon}\right)\right) \mathbf{n}\right\|_{-1 / 2, \Sigma}+\left\|\theta_{D}-W_{\mathscr{C}}\left(\frac{x-z}{\varepsilon}\right)\right\|_{1 / 2, \Gamma}+|\varrho|\right) \\
\leq & c\left(\left\|\theta_{N}\right\|_{-1 / 2, \Sigma}+\left\|\sigma\left(W_{\mathscr{C}}\left(\frac{x-z}{\varepsilon}\right)\right) \mathbf{n}\right\|_{-1 / 2, \Sigma}+\left\|\theta_{D}\right\|_{1 / 2, \Gamma}\right. \\
& \left.+\left\|W_{\mathscr{C}}\left(\frac{x-z}{\varepsilon}\right)\right\|_{1 / 2, \Gamma}+\|\theta\|_{1 / 2, \partial \mathscr{B}_{z, \varepsilon}}\right) .
\end{aligned}
$$

Notice that we obtain, by an adaptation of the same technique used in 4.11:

$$
\left\|\sigma\left(W_{\mathscr{C}}\left(\frac{x-z}{\varepsilon}\right)\right) \mathbf{n}\right\|_{-1 / 2, \Sigma} \leq c\left\|e\left(W_{\mathscr{C}}\right)\left(\frac{x-z}{\varepsilon}\right)\right\|_{0, \Omega_{R}},
$$

where $\Omega_{R}=\Omega \backslash \overline{\mathrm{B}(z, R)}$ with $R>0$ such that $\overline{\mathscr{B}_{z, \varepsilon}} \subset \overline{\mathrm{B}(z, R)} \subset \Omega$.

Using the change of variables $y=(x-z) / \varepsilon$, we get

$$
\begin{aligned}
\left\|e\left(W_{\mathscr{C}}\right)\left(\frac{x-z}{\varepsilon}\right)\right\|_{0, \Omega_{R}} & =\varepsilon\left\|e\left(W_{\mathscr{C}}\left(\frac{x-z}{\varepsilon}\right)\right)\right\|_{0, \Omega_{R}} \\
& =\varepsilon^{2}\left\|e\left(W_{\mathscr{C}}\right)\right\|_{0, \Omega_{R} / \varepsilon} \leq c \varepsilon^{2}\left|W_{\mathscr{C}}\right|_{1, \Omega_{R} / \varepsilon},
\end{aligned}
$$

and from Lemma 7.6 , we have

$$
\left\|\sigma\left(W_{\mathscr{C}}\left(\frac{x-z}{\varepsilon}\right)\right) \mathbf{n}\right\|_{-1 / 2, \Sigma} \leq c \varepsilon^{4}\|\theta\|_{1 / 2, \partial \mathscr{C}}
$$

Now we estimate $\left\|W_{\mathscr{C}}\left(\frac{x-z}{\varepsilon}\right)\right\|_{1 / 2, \Gamma}$. By trace theorem, we obtain

$$
\begin{aligned}
\left\|W_{\mathscr{C}}\left(\frac{x-z}{\varepsilon}\right)\right\|_{1 / 2, \Gamma} & \leq c\left\|W_{\mathscr{C}}\left(\frac{x-z}{\varepsilon}\right)\right\|_{1, \Omega_{R}} \\
& \leq c\left(\left\|W_{\mathscr{C}}\left(\frac{x-z}{\varepsilon}\right)\right\|_{0, \Omega_{R}}+\left\|e\left(W_{\mathscr{C}}\right)\left(\frac{x-z}{\varepsilon}\right)\right\|_{0, \Omega_{R}}\right) .
\end{aligned}
$$

Using the same argument as in (7.17) and we apply $(7.2)$ and $(7.3)$ we deduce that

$$
\left\|W_{\mathscr{C}}\left(\frac{x-z}{\varepsilon}\right)\right\|_{1 / 2, \Gamma} \leq c\left(\varepsilon\|\theta(z+\varepsilon y)\|_{1 / 2, \partial \mathscr{C}}+\varepsilon^{4}\|\theta(z+\varepsilon y)\|_{1 / 2, \partial \mathscr{C}}\right) .
$$

Combining (7.16), (7.17) and (7.18), we have

$$
\begin{aligned}
& \left\|w_{\varepsilon}\right\|_{1, \Omega_{z, \varepsilon}} \\
\leq & c\left(\left\|\theta_{N}\right\|_{-1 / 2, \Sigma}+\left\|\theta_{D}\right\|_{1 / 2, \Gamma}+\varepsilon\|\theta(z+\varepsilon y)\|_{1 / 2, \partial \mathscr{C}}+\varepsilon^{4}\|\theta(z+\varepsilon y)\|_{1 / 2, \partial \mathscr{C}}+\|\theta\|_{1 / 2, \partial \mathscr{B}_{z, \varepsilon}}\right) .
\end{aligned}
$$

Then, for a small enough $\varepsilon$, we get

$$
\left\|w_{\varepsilon}\right\|_{1, \Omega_{z, \varepsilon}} \leq c\left(\left\|\theta_{N}\right\|_{-1 / 2, \Sigma}+\left\|\theta_{D}\right\|_{1 / 2, \Gamma}+\|\theta\|_{1 / 2, \partial \mathscr{B}_{z, \varepsilon}}\right) .
$$




\section{Acknowledgments}

I would like to thank Prof. Maatoug Hassine for his assistance, for many helpful suggestions he made of the manuscript.

\section{References}

[1] A. B. Abda, M. Hassine, M. Jaoua and M. Masmoudi, Topological sensitivity analysis for the location of small cavities in Stokes flow, SIAM J. Control Optim. 48 (2010), no. $5,2871-2900$.

[2] M. Abdelwahed and M. Hassine, Topological optimization method for a geometric control problem in Stokes flow, Appl. Numer. Math. 59 (2009), no. 8, 1823-1838.

[3] G. Alessandrini, A. Bilotta, G. Formica, A. Morassi, E. Rosset and E. Turco, Numerical size estimates of inclusions in elastic bodies, Inverse Problems 21 (2005), no. 1, $133-151$.

[4] G. Alessandrini, A. Morassi and E. Rosset, Detecting an inclusion in an elastic body by boundary measurements, SIAM Rev. 46 (2004), no. 3, 477-498.

[5] G. Allaire, Shape Optimization by the Homogenization Method, Applied Mathematical Sciences 146, Springer-Verlag, New York, 2002.

[6] G. Allaire, F. Jouve and N. Van Goethem, Damage and fracture evolution in brittle materials by shape optimization methods, J. Comput. Phys. 230 (2011), no. 12, 50105044 .

[7] F. Alliot and C. Amrouche, Weak solutions for the exterior Stokes problem in weighted Sobolev spaces, Math. Methods Appl. Sci. 23 (2000), no. 6, 575-600.

[8] C. Alves and H. Ammari, Boundary integral formulae for the reconstruction of imperfections of small diameter in an elastic medium, SIAM J. Appl. Math. 62 (2001), no. 1, 94-106.

[9] C. J. S. Alves and N. F. M. Martins, The direct method of fundamental solutions and the inverse Kirsch-Kress method for the reconstruction of elastic inclusions or cavities, J. Integral Equations Appl. 21 (2009), no. 2, 153-178.

[10] H. Ammari, H. Kang, G. Nakamura and K. Tanuma, Complete asymptotic expansions of solutions of the system of elastostatics in the presence of an inclusion of small diameter and detection of an inclusion, J. Elasticity 67 (2002), no. 2, 97-129. 
[11] S. Amstutz, The topological asymptotic for the Navier-Stokes equations, ESAIM Control Optim. Calc. Var. 11 (2005), no. 3, 401-425.

[12] S. Amstutz and H. Andrä, A new algorithm for topology optimization using a level-set method, J. Comput. Phys. 216 (2006), no. 2, 573-588.

[13] S. Amstutz, I. Horchani and M. Masmoudi, Crack detection by the topological gradient method, Control Cybernet. 34 (2005), no. 1, 81-101.

[14] S. Amstutz and A. A. Novotny, Topological optimization of structures subject to Von Mises stress constraints, Struct. Multidiscip. Optim. 41 (2010), no. 3, 407-420.

[15] D. D. Ang, D. D. Trong and M. Yamamoto, Identification of cavities inside twodimensional heterogeneous isotropic elastic bodies, J. Elasticity 56 (1999), no. 3, 199212.

[16] K. Atkinson and W. Han, Theoretical Numerical Analysis: A functional analysis framework, Third edition, Texts in Applied Mathematics 39, Springer, Dordrecht, 2009.

[17] D. Auroux, M. Masmoudi and L. Belaid, Image restoration and classification by topological asymptotic expansion, in: Variational Formulations in Mechanics: Theory and Applications, 23-42, 2006.

[18] J. B. Bacani and G. Peichl, On the first-order shape derivative of the Kohn-Vogelius cost functional of the Bernoulli problem, Abstr. Appl. Anal. 2013 (2013), Art. ID. 384320, 19 pp.

[19] L. J. Belaid, M. Jaoua, M. Masmoudi and L. Siala, Application of the topological gradient to image restoration and edge detection, Eng. Anal. Bound. Elem. 32 (2008), no. 11, 891-899.

[20] L. M. Bezerra and S. Saigal, A boundary element formulation for the inverse elastostatics problem (IESP) of flaw detection, Internat. J. Numer. Methods Engrg. 36 (1993), no. 13, 2189-2202.

[21] M. Bonnet and A. Constantinescu, Inverse problems in elasticity, Inverse Problems 21 (2005), no. 2, R1-R50.

[22] A. Canelas, A. Laurain and A. A. Novotny, A new reconstruction method for the inverse source problem from partial boundary measurements, Inverse Problems 31 (2015), no. 7, 075009, 24 pp. 
[23] A. Canelas, A. A. Novotny and J. R. Roche, A new method for inverse electromagnetic casting problems based on the topological derivative, J. Comput. Phys. 230 (2011), no. $9,3570-3588$.

[24] F. Caubet, C. Conca and M. Godoy, On the detection of several obstacles in 2D Stokes flow: topological sensitivity and combination with shape derivatives, Inverse Probl. Imaging 10 (2016), no. 2, 327-367.

[25] F. Caubet and M. Dambrine, Localization of small obstacles in Stokes flow, Inverse Problems 28 (2012), no. 10, 105007, 31 pp.

[26] R. Dautray and J.-L. Lions, Mathematical Analysis and Numerical Methods for Science and Technology, Vol. 1: Physical origins and classical methods, Springer-Verlag, Berlin, 1990.

[27] N. Dominguez, V. Gibiat and Y. Esquerre, Time domain topological gradient and time reversal analogy: An inverse method for ultrasonic target detection, Wave Motion 42 (2005), no. 1, 31-52.

[28] K. Eppler and H. Harbrecht, On a Kohn-Vogelius like formulation of free boundary problems, Comput. Optim. Appl. 52 (2012), no. 1, 69-85.

[29] S. Garreau, P. Guillaume and M. Masmoudi, The topological asymptotic for PDE systems: the elasticity case, SIAM J. Control Optim. 39 (2001), no. 6, 1756-1778.

[30] S. M. Giusti, A. A. Novotny and C. Padra, Topological sensitivity analysis of inclusion in two-dimensional linear elasticity, Eng. Anal. Bound. Elem. 32 (2008), no. 11, 926935.

[31] Ph. Guillaume and K. Sid Idris, The topological asymptotic expansion for the Dirichlet problem, SIAM J. Control Optim. 41 (2002), no. 4, 1042-1072.

[32] _ Topological sensitivity and shape optimization for the Stokes equations, SIAM J. Control Optim. 43 (2004), no. 1, 1-31.

[33] M. Hassine and M. Masmoudi, The topological asymptotic expansion for the quasiStokes problem, ESAIM Control Optim. Calc. Var. 10 (2004), no. 4, 478-504.

[34] M. Hintermüller, Fast level set based algorithms using shape and topological sensitivity information, Control Cybernet. 34 (2005), no. 1, 305-324.

[35] M. Hrizi, M. Hassine and R. Malek, A new reconstruction method for a parabolic inverse source problem, Accepted in Appl. Anal., (2018), 13 pp. 
[36] G. C. Hsiao and W. L. Wendland, Boundary Integral Equations, Applied Mathematical Sciences 164, Springer-Verlag, Berlin, 2008.

[37] S.-C. Hsieh and T. Mura, Nondestructive cavity identification in structures, Internat. J. Solids Structures 30 (1993), no. 12, 1579-1587.

[38] K. L. Johnson, The correlation of indentation experiments, J. Mech. Phys. Solids 18 (1970), no. 2, 115-126.

[39] A. Karageorghis, D. Lesnic and L. Marin, The method of fundamental solutions for the detection of rigid inclusions and cavities in plane linear elastic bodies, Computers \& Structures 106-107 (2012), 176-188.

[40] The method of fundamental solutions for three-dimensional inverse geometric elasticity problems, Computers \& Structures 166 (2016), 51-59.

[41] A. J. Kassab, F. A. Moslehy and A. B. Daryapurkar, Nondestructive detection of cavities by an inverse elastostatics boundary element method, Eng. Anal. Bound. Elem. 13 (1994), no. 1, 45-55.

[42] R. V. Kohn and M. Vogelius, Relaxation of a variational method for impedance computed tomography, Comm. Pure Appl. Math. 40 (1987), no. 6, 745-777.

[43] H. S. Lee, Y. H. Kim, C. J. Park and H. W. Park, A new spatial regularization scheme for the identification of the geometric shape of an inclusion in a finite body, Internat. J. Numer. Methods Engrg. 46 (1999), no. 7, 973-992.

[44] L. Marin, L. Elliott, D. B. Ingham and D. Lesnic, Identification of material properties and cavities in two-dimensional linear elasticity, Comput. Mech. 31 (2003), no. 3-4, 293-300.

[45] M. Masmoudi, The topological asymptotic, computational methods for control applications, International Series, Gakuto, 2002.

[46] M. Masmoudi, J. Pommier and B. Samet, The topological asymptotic expansion for the Maxwell equations and some applications, Inverse Problems 21 (2005), no. 2, 547-564.

[47] V. G. Maz'ya and S. V. Poborchi, Differentiable Functions on Bad Domains, World Scientific, 1997.

[48] A. Morassi and E. Rosset, Detecting rigid inclusions, or cavities, in an elastic body, J. Elasticity 73 (2003), no. 1-3, 101-126. 
[49] _ Uniqueness and stability in determining a rigid inclusion in an elastic body, Mem. Amer. Math. Soc. 200 (2009), no. 938, 58 pp.

[50] G. Nakamura and G. Uhlmann, Identification of Lamé parameters by boundary measurements, Amer. J. Math. 115 (1993), no. 5, 1161-1187.

[51] A. A. Novotny and J. Sokołowski, Topological Derivatives in Shape Optimization, Interaction of Mechanics and Mathematics, Springer, Heidelberg, 2013.

[52] G. Rus and R. Gallego, Optimization algorithms for identification inverse problems with the boundary element method, Eng. Anal. Bound. Elem. 26 (2002), no. 4, 315327.

[53] B. Samet, S. Amstutz and M. Masmoudi, The topological asymptotic for the Helmholtz equation, SIAM J. Control Optim. 42 (2003), no. 5, 1523-1544.

[54] A. Schumacher, Topologieoptimierung von Bauteilstrukturen unter Verwendung von Lochpositionierungskriterien, PhD Thesis University of Siegen, 1996.

[55] E. I. Shifrin and P. S. Shushpannikov, Identification of a spheroidal defect in an elastic solid using a reciprocity gap functional, Inverse Problems 26 (2010), no. 5, 055001, $17 \mathrm{pp}$.

[56] J. Sokołowski and A. Żochowski, On the topological derivative in shape optimization, SIAM J. Control Optim. 37 (1999), no. 4, 1251-1272.

[57] A. Wexler, B. Fry and M. R. Neuman, Impedance-computed tomography algorithm and system, Appl. Opt. 24 (1985), no. 23, 3985-3992.

Mourad Hrizi

Monastir University, Department of Mathematics, Faculty of Sciences Avenue de l'Environnement 5000, Monastir, Tunisia

E-mail address: mourad-hrizi@hotmail.fr 